\title{
Effects of Air Pollution on the Risk of Congenital Anomalies: A Systematic Review and Meta-Analysis
}

\author{
Esther Kai-Chieh Chen ${ }^{1}$, Denis Zmirou-Navier ${ }^{1,2,3}$, Cindy Padilla ${ }^{1,2}$ and Séverine Deguen ${ }^{1,2, *}$
}

1 School of Public Health, EHESP, Sorbonne-Paris-Cité, Rennes, Cedex 35043, France;

E-Mails: Esther.Chen@eleve.ehesp.fr (E.K.-C.C.); denis.zmirou@ehesp.fr (D.Z.-N.); cindy.padilla@ehesp.fr (C.P.)

2 Inserm-Unit Research 1085, Research Institute of Environmental and Occupational Health, Rennes, Cedex 35043, France

3 Lorraine University Medical School, Nancy, Cedex 54052, France

* Author to whom correspondence should be addressed; E-Mail: severine.deguen@ehesp.fr; Tel.: +33-299-022-805; Fax: +33-299-022-675.

Received: 26 May 2014; in revised form: 16 July 2014 / Accepted: 16 July 2014 /

Published: 31 July 2014

Abstract: Congenital anomalies are the main causes of preterm and neonatal mortality and morbidity. We investigated the association between congenital anomalies and mothers' exposure to air pollution during pregnancy by combining risk estimates for a variety of air pollutants $\left(\mathrm{SO}_{2}, \mathrm{NO}_{2}, \mathrm{PM}_{10}, \mathrm{PM}_{2.5}, \mathrm{CO}\right.$ and $\left.\mathrm{O}_{3}\right)$ and anomaly defect outcomes. Seventeen articles were included in the systematic review and thirteen studies were taken into account in the meta-analysis. Combined estimated were calculated separately according to whether the exposure metric was continuous or categorical. Only one significant combination was; $\mathrm{NO}_{2}$ concentrations were significantly associated with coarctation of the aorta $(\mathrm{OR}=1.20$ per $10 \mathrm{ppb}, 95 \% \mathrm{CI},(1.02,1.41))$. This finding could stem from strong heterogeneity in study designs. Improved exposure assessment methods, in particular more accurate spatial measurements or modeling, standardized definition of cases and of better control of confounders are highly recommended for future congenital anomalies research in this area.

Keywords: meta-analysis; congenital anomalies; exposure; air pollution 


\section{Introduction}

Today, air pollution is a major public health concern. Due to industrial emissions, urbanization and transport of goods and people by fuel-engine motor vehicles, air pollution affects everyone in developed and developing countries. Common pollutants such as nitrogen oxide $\left(\mathrm{NO}_{2}\right)$, sulfur dioxide $\left(\mathrm{SO}_{2}\right)$, particulate matter (PM) and carbon monoxide (CO) have been shown to be associated with several adverse health events such asthma attacks and incidence [1,2], chronic obstructive pulmonary diseases [3], cardio and cerebrovascular conditions and lung cancer [4-6], and have been reported to reduce human fertility [7]. Environmental nuisances affect all age groups. However, fetus development, newborns and infants are recognized to be more particularly vulnerable to air pollution [8-10]. Exposure of pregnant women is linked with fetal growth retardation, low birth weight [11], preterm birth and neonatal mortality [12]. Maternal exposure to air pollution may be also related to congenital anomalies. However, the evidence is still weak due to the paucity of epidemiological studies.

Congenital anomalies are recognized to be a major risk factor of stillbirth and of neonatal and infant mortality. Worldwide, an estimated $10 \%$ deaths under five-years-old children are caused by congenital anomalies [13]. European Surveillance of Congenital Anomalies (EUROCAT), an organization of population-based registries for the surveillance of congenital anomalies in Europe, recorded the perinatal death rate 9.3 per 10,000 births of all congenital anomaly between 2008 and 2012. Among them, $23.7 \%$ was due to chromosomal anomalies, $22.6 \%$ to congenital heart defects, and $17.2 \%$ to nervous system anomalies [14]. About half of all major congenital malformations are of unclear etiology and are suggested to have multifactorial causes, including environmental exposures [15].

In the past decade, the number of studies investigating the association between congenital anomalies and air pollution has increased. The potential impact of environmental exposures to congenital anomalies has been recently reviewed [16,17]. The studies concluded that exposure to $\mathrm{NO}_{2}$, $\mathrm{SO}_{2}$ and $\mathrm{PM}_{2.5}$ increased significantly the risk of congenital heart diseases. Since the last 2011 review, several new studies have been published [18-23]. In this setting, updating the literature synthesis may improve our understanding of the relationship between air pollution and congenital anomalies and also of the biological process through which air pollution could lead, directly or indirectly, to these outcomes. We, therefore, conducted a meta-analysis to assess the association between air pollution and the risk of congenital anomalies. We explored whether some subtype of anomalies could be particularly concerned by air pollution. Finally, we discussed hypotheses explaining the different routes by which air pollution might increase the risk of congenital anomalies.

\section{Methods}

The methodology adopted has been described in detail in the previous published review [16]. Meta-analyses were conducted for a minimum number of four individual studies. We separated the data set into two categories according to the metric used for exposure assessment (continuous or categorical). Combined odds ratios were computed in order to contrast the highest with the lowest quartiles of exposure when the individual studies reported categorical exposure metrics. When exposure was expressed as a continuous variable, if quantitative descriptors for air pollutants were available, we also converted effect estimates into ORs contrasting the highest versus the lowest exposure categories [24]. 
Otherwise, risk estimates which had been calculated from continuous exposure metrics were expressed as unit odds ratios, corresponding to an increase of 1 microgram per cubic meter $\left(\mu \mathrm{g} / \mathrm{m}^{3}\right)$ for sulfur dioxide $\left(\mathrm{SO}_{2}\right), 10 \mu \mathrm{g} / \mathrm{m}^{3}$ for particulate matter with diameter less than $10 \mu \mathrm{m}\left(\mathrm{PM}_{10}\right)$ and to an increase of 1 part per million (ppm) for carbon oxide (CO), and 5 part per billion (ppb) for ozone $\left(\mathrm{O}_{3}\right)$ and $10 \mathrm{ppb}$ nitrogen dioxide $\left(\mathrm{NO}_{2}\right)$. Key features and definitions of exposure of each study are detailed in Table 1.

\subsection{Search Methods}

A literature search was conducted in the PUBMED database in order to select articles published between January 2011 and January 2014. The search strategy followed the PRISMA guidelines [25]. The keywords used for this review were (air pollution OR traffic pollution OR outdoor pollution) AND congenital anomalies. We also used the terms "traffic pollution" and "outdoor air pollution". Searches were restricted to English-language articles. No restriction was put on the geographical location. Abstracts of all studies were then screened manually and excluded if they were not performed on human populations and did not present original data (review articles). Full manuscripts were checked thoroughly. Seven studies were published after 2011; one was not included because its main issue did not deal with the association between congenital anomalies and air pollutants [26]. We also included the eleven articles used in the previous literature synthesis published in 2011. Overall, seventeen articles were included.

\subsection{Data Extraction}

We selected measures from the adjusted models presented in each study. Odds ratios and similar metrics relating outcomes and pollutants were extracted. For cohort studies, we used risk ratios since the two ratios give equivalent results when the outcome is rare. In addition, the period of exposure during the pregnancy has been taken into account since the pregnancy weeks 3-8 constitute the critical window of exposure for embryogenesis; later exposures may not contribute to the etiology of major congenital anomalies [22].

\subsection{Meta-Analysis}

Heterogeneity was assessed for pollutant-congenital anomaly outcomes by using the Cochrane Q-test. Fixed effect models were used when the result of the Q-test gave a heterogeneity $p$-value higher than 0.1. In contrast, random effects models were used for p-values lower than 0.1. Following Higgins et al. [27], a low heterogeneity was determined for $\mathrm{I}^{2}$ between $25 \%-50 \%$, moderate between $50 \%-75 \%$, and high for $>75 \%$, where $\mathrm{I}^{2}$ is defined as the percentage of variation attributable to heterogeneity. Forest plots were generated to illustrate the combined risk estimates. Statistical analysis was performed using STATA 11 (TX, USA). 
Table 1. Overview of studies included in the systematic review.

\begin{tabular}{|c|c|c|c|c|c|c|c|c|c|}
\hline Study & Location & Period & Study Design & Congenital Categories & Exposure Assessment & Exposure Variable & Air Pollutants & Results & Confounders \\
\hline $\begin{array}{c}\text { Gianicolo } \\
\text { et al. } 2014 \\
{[23]}\end{array}$ & Brindisi, Italy & $2000-2010$ & $\begin{array}{l}\text { Case-control, } \\
\text { individual } \\
\text { matching }\end{array}$ & $\begin{array}{c}\text { Congenital heart defects, } \\
\text { atrial septal defects }\end{array}$ & $\begin{array}{c}\text { Daily average } \\
\text { concentration of } \\
\text { pollutants measured by } 3 \\
\text { monitoring stations and } \\
\text { performed for week } 3-8 \\
\text { of gestation }\end{array}$ & $\begin{array}{c}\text { Continuous and } \\
\text { categorical }\end{array}$ & $\mathrm{SO}_{2}$ and TSP & $\begin{array}{c}\text { Exposure to } 90 \text { th } \\
\text { percentile of } \mathrm{SO}_{2} \\
\text { increased risk of CHD } \\
(p=0.01) \text { and VSD } \\
(p<0.05)\end{array}$ & $\begin{array}{c}\text { No adjusted } \\
\text { confounders; cases } \\
\text { and controls were } \\
\text { matching for gender, } \\
\text { socio-economic } \\
\text { deprivation and the } \\
\text { year of pregnancy }\end{array}$ \\
\hline $\begin{array}{l}\text { Schembari } \\
\text { et al. } 2013 \\
{[22]}\end{array}$ & $\begin{array}{l}\text { Barcelona, } \\
\text { Spain }\end{array}$ & 1994-2006 & $\begin{array}{l}\text { Case-control, } \\
\text { no matching }\end{array}$ & $\begin{array}{l}\text { Congenital heart defects, } \\
\text { neural tube defects, } \\
\text { respiratory system } \\
\text { defects, orofacial clefts, } \\
\text { digestive system defects, } \\
\text { abdominal wall }\end{array}$ & $\begin{array}{l}\text { Daily spatio-temperal } \\
\text { exposure estimates over } \\
\text { week } 3-8 \text { of pregnancy }\end{array}$ & Continuous & $\begin{array}{c}\mathrm{NO}_{2}, \mathrm{NO}_{\mathrm{x}}, \mathrm{PM}_{10} \\
\mathrm{PM}_{2.5}, \mathrm{PM}_{\text {coarse }}\end{array}$ & $\begin{array}{l}\text { Statistically significant } \\
\text { associations }(p=0.05) \\
\text { between } \mathrm{NO}_{2} \text { and } \\
\text { coarctation of the aorta } \\
\text { and digestive system } \\
\text { defects, and between } \\
\mathrm{PM}_{\text {coarse }} \text { and abdominal } \\
\text { wall defects }\end{array}$ & $\begin{array}{c}\text { Maternal age, } \\
\text { socio-economic } \\
\text { status, year of birth, } \\
\text { conception season }\end{array}$ \\
\hline $\begin{array}{l}\text { Padula et al., } \\
\qquad \begin{array}{l}2013 \\
{[20]}\end{array}\end{array}$ & $\begin{array}{c}\text { California, } \\
\text { USA }\end{array}$ & 1997-2006 & $\begin{array}{l}\text { Case-control, } \\
\text { no matching }\end{array}$ & $\begin{array}{c}\text { Anotia/microtia, } \\
\text { anorectal atresia/stenosis, } \\
\text { craniosynostosis, hypospadias } \\
\text { degree, diaphragmatic hernia, } \\
\text { transverse limb deficiency, } \\
\text { intestinal atresia/stenosis, } \\
\text { amniotic band syndrome, } \\
\text { limb body wall complex, } \\
\text { hydrocephaly, } \\
\text { longitudinal limb deficiency, } \\
\text { esophageal atresia }\end{array}$ & $\begin{array}{l}\text { Residence-based } \\
\text { assignments around } \\
\text { stations, with daily } \\
\text { average values during } \\
\text { first two months of were } \\
\text { collected; a maximum } \\
\text { interpolation radius } \\
\text { of } 50 \text { km was used } \\
\end{array}$ & Categorical & $\begin{array}{c}\mathrm{NO}_{2}, \mathrm{NO}, \mathrm{CO} \\
\mathrm{O}_{3}, \mathrm{PM}_{10}, \mathrm{PM}_{2.5}\end{array}$ & $\begin{array}{c}\text { No significant } \\
\text { association had been } \\
\text { revealed }\end{array}$ & $\begin{array}{l}\text { Maternal ethnicity, } \\
\text { education, and early } \\
\text { prenatal vitamin use }\end{array}$ \\
\hline
\end{tabular}


Table 1. Cont

\begin{tabular}{|c|c|c|c|c|c|c|c|c|c|}
\hline Study & Location & Period & Study Design & Congenital Categories & Exposure Assessment & $\begin{array}{l}\text { Exposure } \\
\text { Variable }\end{array}$ & Air Pollutants & Results & Confounders \\
\hline $\begin{array}{c}\text { Padula } \\
\text { et al. } 2013 \\
{[19]}\end{array}$ & $\begin{array}{c}\text { California, } \\
\text { USA }\end{array}$ & 1997-2006 & $\begin{array}{l}\text { Case-control, } \\
\text { no matching }\end{array}$ & $\begin{array}{l}\text { Congenital heart diseases } \\
\text { groups ( } 27 \text { subtypes) }\end{array}$ & $\begin{array}{c}\text { Residence-based } \\
\text { assignments around stations, } \\
\text { with daily average values } \\
\text { during first two months of } \\
\text { were collected; a maximum } \\
\text { interpolation radius } \\
\text { of } 50 \mathrm{~km} \text { was used }\end{array}$ & Categorical & $\begin{array}{c}\mathrm{NO}, \mathrm{NO}_{2}, \mathrm{PM}_{10}, \\
\mathrm{PM}_{2.5}, \mathrm{CO}, \mathrm{O}_{3}\end{array}$ & $\begin{array}{c}\text { No significant } \\
\text { association had been } \\
\text { revealed }\end{array}$ & $\begin{array}{c}\text { Maternal } \\
\text { ethnicity, education, } \\
\text { and vitamin use }\end{array}$ \\
\hline $\begin{array}{l}\text { Agay-Shay } \\
\text { K et al. } \\
2013 \text { [21] }\end{array}$ & $\begin{array}{c}\text { Tel-Aviv, } \\
\text { Israel }\end{array}$ & 2000-2006 & $\begin{array}{l}\text { Case-control, } \\
\text { no matching }\end{array}$ & $\begin{array}{l}\text { Multiple congenital heart, } \\
\text { atrial and atrial } \\
\text { septal defects, } \\
\text { isolated ventricular } \\
\text { septal defects }\end{array}$ & $\begin{array}{l}\text { Weekly means of exposures } \\
\text { during pregnancy week } 3-8 \\
\text { according to the distance from } \\
\text { stations to each maternal } \\
\text { address }\end{array}$ & Continuous & $\begin{array}{c}\mathrm{NO}_{2}, \mathrm{SO}_{2}, \\
\mathrm{PM}_{10}, \mathrm{PM}_{2.5} \\
\mathrm{CO}, \mathrm{O}_{3}\end{array}$ & $\begin{array}{c}\text { No significant } \\
\text { association had been } \\
\text { revealed }\end{array}$ & $\begin{array}{l}\text { Infant's sex, } \\
\text { plurality, religion, } \\
\text { maternal age, } \\
\text { maternal and paternal } \\
\text { marital status, } \\
\text { maternal and } \\
\text { paternal origin, } \\
\text { paternal age, and the } \\
\text { season of conception }\end{array}$ \\
\hline $\begin{array}{c}\text { Padula } \\
\text { et al. } 2013 \\
{[18]}\end{array}$ & $\begin{array}{c}\text { California, } \\
\text { USA }\end{array}$ & 1997-2006 & $\begin{array}{l}\text { Case-control, } \\
\text { no matching }\end{array}$ & $\begin{array}{l}\text { Neural tubes defects (spina } \\
\text { bifida and anencephaly), } \\
\text { orofacial clefts, gastroschisis }\end{array}$ & $\begin{array}{l}\text { Residence-based assignments } \\
\text { around stations, with daily } \\
\text { average values during first two } \\
\text { months of were collected; } \\
\text { a maximum interpolation radius } \\
\text { of } 50 \mathrm{~km} \text { was used }\end{array}$ & Categorical & $\begin{array}{c}\mathrm{NO}_{2}, \mathrm{NO}, \\
\mathrm{PM}_{10}, \mathrm{PM}_{2.5}, \\
\mathrm{CO}, \mathrm{O}_{3}\end{array}$ & $\begin{array}{c}\text { No significant } \\
\text { association had been } \\
\text { revealed }\end{array}$ & $\begin{array}{c}\text { Maternal ethnicity, } \\
\text { education, and } \\
\text { vitamin use }\end{array}$ \\
\hline
\end{tabular}


Table 1. Cont

\begin{tabular}{|c|c|c|c|c|c|c|c|c|c|}
\hline Study & Location & Period & Study Design & Congenital Categories & Exposure Assessment & Exposure Variable & Air Pollutants & Results & Confounders \\
\hline $\begin{array}{c}\text { Dadvand } \\
\text { et al. } 2011 \\
{[28]}\end{array}$ & $\begin{array}{c}\text { Northeast of } \\
\text { UK }\end{array}$ & 1993-2003 & $\begin{array}{l}\text { Case-control, } \\
\text { frequency } \\
\text { matching }\end{array}$ & $\begin{array}{c}\text { Cardiac chambers and } \\
\text { connection, cardiac septa, } \\
\text { pulmonary and tricupid } \\
\text { valves, aortic and mitral } \\
\text { valves, great arteries and } \\
\text { veins, atrial septal defect, } \\
\text { coarctation of aorta, } \\
\text { pulmonary valve stenosis, } \\
\text { tetralogy of Fallot, } \\
\text { ventricular septal defect }\end{array}$ & $\begin{array}{l}\text { Weekly average of } \\
\text { pollutants at nearest } \\
\text { monitors to maternal } \\
\text { residential location }\end{array}$ & Continuous & $\begin{array}{c}\mathrm{SO}_{2}, \mathrm{NO}_{2}, \mathrm{CO} \\
\mathrm{PM}_{10}, \mathrm{O}_{3}\end{array}$ & $\begin{array}{c}\text { An association between } \\
\mathrm{NO}_{2} \text { and congenital heart } \\
\text { diseases, ventricular septal } \\
\text { defect, cardiac septa } \\
\text { malformations and } \\
\text { tetralogy of Fallot; and } \\
\mathrm{CO} \text { exposure to } \\
\text { ventricular septal defect, } \\
\text { cardiac septa } \\
\text { malformations and with } \\
\text { congenital pulmonary } \\
\text { valve stenosis }\end{array}$ & $\begin{array}{l}\text { Socio-economic } \\
\text { status, degree of } \\
\text { urbanity, and season } \\
\text { of conception; } \\
\text { cases and controls } \\
\text { were matching for } \\
\text { the year of birth }\end{array}$ \\
\hline $\begin{array}{c}\text { Dadvand } \\
\text { et al. } 2011 \\
\text { [29] }\end{array}$ & $\begin{array}{c}\text { Northeast of } \\
\text { UK }\end{array}$ & 1985-1996 & $\begin{array}{l}\text { Case-control, } \\
\text { frequency } \\
\text { matching }\end{array}$ & $\begin{array}{l}\text { Coarctation of aorta, } \\
\text { tetralogy of fallot, congenital } \\
\text { pulmonary value stenosis, } \\
\text { atrial septal defect, } \\
\text { ventricular septal defect, } \\
\text { congenital cardiac chambers } \\
\text { and connections, congenital } \\
\text { cardia septa, congenital } \\
\text { pulmonary and tricuspid } \\
\text { valves, congenital aortic and } \\
\text { mitral valves, congenital great } \\
\text { arteries and veins }\end{array}$ & $\begin{array}{c}\text { Weekly exposure } \\
\text { levels by two stage } \\
\text { spaiotemporal modeling } \\
\text { at each maternal place } \\
\text { of residence }\end{array}$ & $\begin{array}{c}\text { Categorical and } \\
\text { continuous }\end{array}$ & $\begin{array}{c}\mathrm{SO}_{2}, \\
\text { black smoke }\end{array}$ & $\begin{array}{l}\text { An association between } \\
\text { maternal exposure to } \\
\text { black smoke and cardiac } \\
\text { chambers and } \\
\text { connections (only when } \\
\text { using exposure as a } \\
\text { continuous variable) }\end{array}$ & $\begin{array}{c}\text { Birth year, } \\
\text { socio-economic } \\
\text { status, infant sex, } \\
\text { season of conception, } \\
\text { and degree of } \\
\text { urbanity; cases and } \\
\text { controls were } \\
\text { matching for the } \\
\text { year of birth }\end{array}$ \\
\hline
\end{tabular}


Table 1. Cont.

\begin{tabular}{|c|c|c|c|c|c|c|c|c|c|}
\hline Study & Location & Period & Study Design & $\begin{array}{l}\text { Congenital } \\
\text { Categories }\end{array}$ & Exposure Assessment & $\begin{array}{l}\text { Exposure } \\
\text { Variable }\end{array}$ & Air Pollutants & Results & Confounders \\
\hline $\begin{array}{l}\text { Marshall } \\
\text { et al. } 2010 \\
\text { [30] }\end{array}$ & $\begin{array}{c}\text { New Jersey, } \\
\text { US }\end{array}$ & $1998-2003$ & $\begin{array}{l}\text { Case-control, } \\
\text { frequency } \\
\text { matching }\end{array}$ & $\begin{array}{c}\text { Cleft lip with cleft palate, } \\
\text { cleft palate }\end{array}$ & $\begin{array}{l}\text { Average concentration } \\
\text { of exposures at nearest } \\
\text { monitor stations } \\
\qquad(13-20 \mathrm{~km})\end{array}$ & Categorical & $\begin{array}{l}\mathrm{SO}_{2}, \mathrm{NO}_{2}, \mathrm{CO} \\
\mathrm{PM}_{10}, \mathrm{PM}_{2.5}, \mathrm{O}_{3}\end{array}$ & $\begin{array}{c}\text { No significant } \\
\text { association had been } \\
\text { revealed }\end{array}$ & $\begin{array}{c}\text { Maternal age, } \\
\text { ethnicity, smoking } \\
\text { and alcohol, and } \\
\text { season of conception; } \\
\text { cases and controls } \\
\text { were matching with } \\
\text { maternal residence at } \\
\text { birth }\end{array}$ \\
\hline $\begin{array}{c}\text { Dolk } \\
\text { et al. } 2010 \\
{[31]}\end{array}$ & $\begin{array}{l}\text { Wessex, } \\
\text { North West } \\
\text { Thamas, } \\
\text { Oxford and } \\
\text { Northern of } \\
\text { UK }\end{array}$ & d $1991-1999$ & Cohort & $\begin{array}{c}\text { Anomalies of cardiac } \\
\text { chambers, transposition of } \\
\text { great vessels, } \\
\text { malformations of cardiac } \\
\text { septa, atrioventricular } \\
\text { septal defects, tetralogy of } \\
\text { Fallot, malformations of } \\
\text { valves, hypoplastice left } \\
\text { heart syndrome, great } \\
\text { arteries and veins, } \\
\text { coarctation of aorta }\end{array}$ & $\begin{array}{c}\text { Annual mean } \\
\text { exposure at census } \\
\text { level in } 1996\end{array}$ & Continuous & $\begin{array}{l}\mathrm{SO}_{2}, \mathrm{NO}_{2} \\
\mathrm{PM}_{10}\end{array}$ & $\begin{array}{c}\text { A significant } \\
\text { association between } \\
\mathrm{SO}_{2} \text { and tetralogy of } \\
\text { Fallot, and between } \\
\mathrm{PM}_{10} \text { and omphalocele }\end{array}$ & $\begin{array}{c}\text { Maternal age, } \\
\text { socio-economic } \\
\text { deprivation }\end{array}$ \\
\hline
\end{tabular}


Table 1. Cont

\begin{tabular}{|c|c|c|c|c|c|c|c|c|c|}
\hline Study & Location & Period & Study Design & Congenital Categories & Exposure Assessment & Exposure Variable & Air Pollutants & Results & Confounders \\
\hline $\begin{array}{c}\text { Hansen } \\
\text { et al. } 2009 \\
\text { [32] }\end{array}$ & $\begin{array}{l}\text { Brisbane, } \\
\text { Australia }\end{array}$ & 1998-2004 & $\begin{array}{l}\text { Case-control, } \\
\text { individual } \\
\text { matching }\end{array}$ & $\begin{array}{l}\text { Aortic artery and valve } \\
\text { defects, atrial septal } \\
\text { defects, pulmonary artery } \\
\text { and valve defects, } \\
\text { ventricular septal defects, } \\
\text { conotruncal defects, } \\
\text { endocardial cushion and } \\
\text { mitral valve defects, cleft } \\
\text { lip, cleft palate, cleft lip } \\
\text { with cleft palate }\end{array}$ & $\begin{array}{l}\text { Daily average exposures } \\
\text { at } 18 \text { monitors with the } \\
\text { majority located within } \\
\text { a } 30 \mathrm{~km} \text { radius of city }\end{array}$ & Continuous & $\begin{array}{l}\mathrm{SO}_{2}, \mathrm{NO}, \mathrm{CO}, \\
\mathrm{PM}_{10} \text { and } \mathrm{O}_{3}\end{array}$ & $\begin{array}{c}\text { No significant association } \\
\text { had been revealed }\end{array}$ & $\begin{array}{l}\text { Infant sex, birth order, } \\
\text { season of birth, } \\
\text { maternal age, } \\
\text { education, alcohol, } \\
\text { and body mass index; } \\
\text { cases and controls } \\
\text { were matching with } \\
\text { mother's age, } \\
\text { marital status, } \\
\text { number of previous } \\
\text { pregnancies, } \\
\text { month of LMP, } \\
\text { area-level SES, } \\
\text { and distance to } \\
\text { pollution monitor }\end{array}$ \\
\hline $\begin{array}{c}\text { Rankin } \\
\text { et al., } 2009 \\
{[33]}\end{array}$ & $\begin{array}{l}\text { Northern } \\
\text { region, UK }\end{array}$ & 1985-1990 & $\begin{array}{l}\text { Case-control, } \\
\text { no matching }\end{array}$ & $\begin{array}{l}\text { Nervous system, } \\
\text { congenital heart defects, } \\
\text { atrio ventricular septal } \\
\text { defects, tetralogy of fallot, } \\
\text { hypoplastic left heart, } \\
\text { coarctation of aorta, patent } \\
\text { ductus arteriosus, } \\
\text { ventricular septal defect, } \\
\text { respiratory tract, cleft lip } \\
\text { and palate, eye, ear, face } \\
\text { and neck, digestive } \\
\text { system, internal urogenital } \\
\text { system, musculoskeletal, } \\
\text { miscellaneous }\end{array}$ & $\begin{array}{l}\text { Daily average exposures } \\
\text { during the first trimester } \\
\text { from monitors within } 10 \\
\mathrm{~km} \text { of maternal residence }\end{array}$ & $\begin{array}{c}\text { Continuous and } \\
\text { categorical }\end{array}$ & SO2, black smoke & $\begin{array}{l}\text { A significant association } \\
\text { between black smoke and } \\
\text { nervous system } \\
\text { anomalies. }\end{array}$ & $\begin{array}{l}\text { Birth weight, infant } \\
\text { sex, and maternal } \\
\text { deprivation }\end{array}$ \\
\hline
\end{tabular}


Table 1. Cont

\begin{tabular}{|c|c|c|c|c|c|c|c|c|c|}
\hline Study & Location & Period & Study Design & Congenital Categories & Exposure Assessment & $\begin{array}{l}\text { Exposure } \\
\text { Variable }\end{array}$ & Air Pollutants & Results & Confounders \\
\hline $\begin{array}{c}\text { Strickland } \\
\text { et al. } 2009 \\
{[34]}\end{array}$ & $\begin{array}{l}\text { Atlanta, } \\
\text { USA }\end{array}$ & 1986-2003 & Cohort & $\begin{array}{l}\text { Tansposition of the great } \\
\text { arteries, tetralogy of fallot, } \\
\text { pulmonary stenosis and valvar, } \\
\text { patent ductus arteriosus, } \\
\text { hypoplastic left heart, } \\
\text { coarctation of aorta, } \\
\text { atrial septal defect, secundum, } \\
\text { ventricular septal defect, } \\
\text { muscular, ventricular septal } \\
\text { defect, permimemnranous, } \\
\text { conotruncal defect, } \\
\text { Left ventricular outflow tract } \\
\text { defect, right ventricular outflow } \\
\text { tract defect }\end{array}$ & $\begin{array}{l}\text { Average of daily } \\
\text { concentration from } \\
\text { one central monitoring } \\
\text { station }\end{array}$ & Continuous & $\begin{array}{c}\mathrm{SO}_{2}, \mathrm{NO}_{2}, \mathrm{CO} \\
\mathrm{PM}_{10} \text {, and } \mathrm{O}_{3}\end{array}$ & $\begin{array}{l}\text { A significant association } \\
\text { between } \mathrm{PM}_{10} \text { and patent } \\
\text { ductus arteriosus }\end{array}$ & $\begin{array}{l}\text { No adjusted } \\
\text { variables }\end{array}$ \\
\hline $\begin{array}{c}\text { Hwang } \\
\text { et al. } 2008 \\
{[24]}\end{array}$ & Taiwan & $2001-2003$ & $\begin{array}{l}\text { Case-control, } \\
\text { no matching }\end{array}$ & Cleft lip & $\begin{array}{l}\text { Monthly average of } \\
\text { exposures at } 72 \text { stations } \\
\text { by using inverse } \\
\text { distance weighting } \\
\text { method during the } \\
\text { first trimester }\end{array}$ & Continuous & $\begin{array}{c}\mathrm{SO}_{2}, \mathrm{NO}_{2}, \mathrm{CO} \\
\mathrm{PM}_{10} \text { and } \mathrm{O}_{3}\end{array}$ & $\begin{array}{l}\text { A significant association } \\
\text { for first and second month } \\
\qquad \mathrm{O}_{3} \text { exposure }\end{array}$ & $\begin{array}{l}\text { Maternal age, infant } \\
\text { sex, plurality }{ }^{\S} \\
\text { gestational age, } \\
\text { population density, } \\
\text { and season of } \\
\text { conception }\end{array}$ \\
\hline
\end{tabular}


Table 1. Cont.

\begin{tabular}{|c|c|c|c|c|c|c|c|c|c|}
\hline Study & Location & Period & $\begin{array}{l}\text { Study } \\
\text { Design }\end{array}$ & $\begin{array}{l}\text { Congenital } \\
\text { Categories }\end{array}$ & Exposure Assessment & $\begin{array}{l}\text { Exposure } \\
\text { Variable }\end{array}$ & Air Pollutants & Results & Confounders \\
\hline $\begin{array}{c}\text { Kim } \\
\text { et al. } 2007 \\
{[35]}\end{array}$ & $\begin{array}{l}\text { Seoul, } \\
\text { Korea }\end{array}$ & 2001-2004 & Birth cohort & Not specified & $\begin{array}{l}\text { Residence based average } \\
\text { exposure levels at each } \\
\text { trimester from nearest } \\
\text { monitoring stations }\end{array}$ & $\begin{array}{c}\text { Continuous and } \\
\text { categorical }\end{array}$ & $\mathrm{PM}_{10}$ & $\begin{array}{c}\text { Congenital anomalies } \\
\text { were influenced by } \\
\text { exposure to } \mathrm{PM}_{10}\end{array}$ & $\begin{array}{l}\text { Infant sex, birth order, } \\
\text { season of birth, maternal } \\
\text { age, maternal and paternal } \\
\text { education, alcohol, body } \\
\text { mass index and maternal } \\
\text { weight before delivery }\end{array}$ \\
\hline $\begin{array}{c}\text { Giloba } \\
\text { et al. } 2005 \\
{[36]}\end{array}$ & $\begin{array}{c}\text { Texas, } \\
\text { USA }\end{array}$ & $1997-2000$ & $\begin{array}{l}\text { Case-control, } \\
\text { frequency } \\
\text { matching }\end{array}$ & $\begin{array}{c}\text { Aortic artery and } \\
\text { valve defects, atrial } \\
\text { septal defects, } \\
\text { pulmonary artery and } \\
\text { valve defects, } \\
\text { ventricular septal } \\
\text { defects, conotruncal } \\
\text { defects, endo-cardial } \\
\text { cushin and mitral } \\
\text { valve defects, cleft lip } \\
\text { with cleft palate, cleft } \\
\text { palate }\end{array}$ & $\begin{array}{l}\text { Average of daily } \\
\text { measurements based on } \\
\text { the first closest monitor } \\
\text { (median distance } \\
\quad 8.6-14.2 \mathrm{~km} \text { ) }\end{array}$ & Categorical & $\begin{array}{c}\mathrm{SO}_{2}, \mathrm{NO}_{2}, \mathrm{CO} \\
\mathrm{PM}_{10} \text { and } \mathrm{O}_{3}\end{array}$ & $\begin{array}{c}\text { A significant association } \\
\text { between exposure to } \mathrm{SO}_{2} \\
\text { and } \mathrm{VSD}(p<0.0001), \\
\mathrm{CO} \text { and tetralogy of fallot } \\
(p<0.0017), \mathrm{PM}_{10} \text { and ASD } \\
(p<0.0001), \mathrm{SO}_{2} \text { with ASD } \\
(0.0017)\end{array}$ & $\begin{array}{c}\text { Maternal age, ethnicity, } \\
\text { education, marital status, } \\
\text { illness, tobacco use, } \\
\text { season }{ }^{\S} \text { of conception, } \\
\text { plurality, parity, infant sex, } \\
\text { prenatal care, } \\
\text { and gravidity; cases and } \\
\text { control were matching with } \\
\text { vital status, year, } \\
\text { maternal county of } \\
\text { residence at delivery }\end{array}$ \\
\hline
\end{tabular}


Table 1. Cont.

\begin{tabular}{|c|c|c|c|c|c|c|c|c|c|}
\hline Study & Location & Period & Study Design & Congenital Categories & Exposure Assessment & Exposure Variable & Air Pollutants & Results & Confounders \\
\hline $\begin{array}{c}\text { Ritz } \\
\text { et al. } 2002 \\
\text { [9] }\end{array}$ & $\begin{array}{c}\text { California, } \\
\text { USA }\end{array}$ & 1987-1993 & $\begin{array}{l}\text { Case-control, } \\
\text { no matching }\end{array}$ & $\begin{array}{c}\text { Aortic defects, } \\
\text { Pulmonary valve, } \\
\text { Conotruncal defects, } \\
\text { Ventricular septal defects, } \\
\text { Multiple cardiac or } \\
\text { cleft defect, Syndrome } \\
\text { with cardiac or cleft } \\
\text { defect, Isolated cleft } \\
\text { palate, Isolated cleft lip } \\
\text { with/without palate }\end{array}$ & $\begin{array}{c}24 \mathrm{~h} \text { average } \\
\text { measurements every } \\
6 \text { days over duration of } \\
\text { pregnancy }\end{array}$ & $\begin{array}{c}\text { Continuous and } \\
\text { categorical }\end{array}$ & $\mathrm{CO}, \mathrm{O}_{3}$ & $\begin{array}{c}\text { No significant } \\
\text { association had been } \\
\text { revealed }\end{array}$ & $\begin{array}{l}\text { Maternal age, ethnicity, } \\
\text { education, marital status, } \\
\text { illness, tobacco use, } \\
\text { season of conception, } \\
\text { plurality }{ }^{\S} \text {, parity, } \\
\text { decade of infant's birth, } \\
\text { infant sex, access to } \\
\text { prenatal care, } \\
\text { time since last pregnancy } \\
\text { and birth type }\end{array}$ \\
\hline
\end{tabular}

Notes: $\mathrm{SO}_{2}$, sodium dioxide; $\mathrm{NO}$, nitrogen oxide; $\mathrm{CO}$, carbon oxide; $\mathrm{PM}_{10}$, particulate meter with diameter $\leq 10 \mu \mathrm{m} ; \mathrm{PM}_{2.5}$, particulate meter with diameter $\leq 2.5 \mu \mathrm{m}$; $\mathrm{O}_{3}$, ozone; TSP, total suspended particulate; CHD, congenital heart defects; ASD, atrial septal defects. $\$$ Plurality stands for multiple births (including stillbirth) after one pregnancy. 


\section{Results}

Seventeen articles met the inclusion criteria for the systematic review, and the characteristics of each study are shown in Table 1. Three articles published in 2013 were conducted by the same research group [18-20]. We considered them as three independent studies because each dealt with different outcomes. Another team also split results into two articles [28,29]. Seven studies were conducted in the United States [9,18-20,30,34,36], four in the United Kingdom [28,29,31,33] and only one in Australia [32], in Israel [21], in Italy [23], in South Korea [35], in Spain [22] and in Taiwan [24]. Overall, twelve studies were case-control studies and five were cohort studies. One did not specify congenital subgroups and diagnosed only birth defects $(n=14)$; it was not included in the meta analysis [35].

Most studies used a population-based case-control design, selecting cases from clinical or autopsy reports. Controls were randomly selected from birth registries. Cases included live birth, stillbirth or termination of pregnancy after a congenital anomaly diagnosis. One study collected only newborns with diagnosis of congenital anomalies [23]. Two studies focused specifically on orofacial defects [24,30] and therefore included only cases with a diagnosis of cleft lip with or without cleft palate. Definitions of cases with single or multiple congenital defects and criteria for splitting them into sub-groups varied across studies. Cases definition used in the studies published after 2010 were mainly coded according to the International Classification of Diseases, version 9 or 10 (ICD codes). Cardiovascular anomalies were the most frequently investigated defects, followed by neuron system.

Mother residence concentration estimates from air quality monitoring networks were frequently used for exposure assessment. Average concentrations of pollutants during the first or the first two months of pregnancy were calculated from the nearest monitors. The distance from monitors to maternal residence varied among studies, from $10 \mathrm{~km}$ to a maximum of $50 \mathrm{~km}$. Classical air pollutants were measured, i.e., $\mathrm{SO}_{2}, \mathrm{NO}_{2}, \mathrm{PM}_{10}, \mathrm{PM}_{2.5}, \mathrm{CO}$ and $\mathrm{O}_{3}$ in most studies. Total suspended particulate (TSP), NO, $\mathrm{NO}_{\mathrm{x}}$ and black smoke were assessed in a small number of studies. Pollutant concentration distributions are shown in Table 2.

We conducted the meta-analysis for 21 combinations of air pollutants and congenital anomalies when at least four studies were available for the same combination. Heterogeneity tests (the Q-test) indicated four combinations with high values of $\mathrm{I}^{2}$, for which random effects models were applied. Heterogeneity varied between $0 \%$ and $93.4 \%$, which indicated that the measurement methods, sample property and characteristics varied among and within different congenital groups. 
Table 2. Exposure distribution in studies included in the meta analysis.

\begin{tabular}{|c|c|c|c|c|c|}
\hline \multirow{2}{*}{ Studies } & \multicolumn{2}{|c|}{ Case } & \multicolumn{2}{|c|}{ Control } & \multirow{2}{*}{ Total } \\
\hline & Congenital Defects & Air Pollutants & Congenital Defects & Air Pollutants & \\
\hline \multirow{2}{*}{$\begin{array}{l}\text { Gianicolo et al. } \\
2014 \text { [23] }\end{array}$} & CHD & $\mathrm{SO}_{2}$, mean $2.9 \mu \mathrm{g} / \mathrm{m}^{3}$ & CHD & $\mathrm{SO}_{2}$, mean $2.8 \mu \mathrm{g} / \mathrm{m}^{3}$ & \multirow[b]{2}{*}{ - } \\
\hline & VSD & $\mathrm{SO}_{2}$, mean $3.2 \mu \mathrm{g} / \mathrm{m}^{3}$ & VSD & $\mathrm{SO}_{2}$, mean $2.8 \mu \mathrm{g} / \mathrm{m}^{3}$ & \\
\hline Schembari et al. & - & IQR: $\mathrm{NO}_{2}, 12.7 \mu \mathrm{g} / \mathrm{m}^{3}$ & - & $\mathrm{NO}_{2}, \mathrm{IQR} 11.8 \mu \mathrm{g} / \mathrm{m}^{3}$ & \multirow[b]{2}{*}{ - } \\
\hline $2013[22]$ & - & $\mathrm{PM}_{10}, 2.8 \mu \mathrm{g} / \mathrm{m}^{3}$ & - & $\mathrm{PM}_{10}, \mathrm{IQR} 3.0 \mu \mathrm{g} / \mathrm{m}^{3}$ & \\
\hline $\begin{array}{l}\text { Agay-Shay et al. } \\
2013 \text { [21] }\end{array}$ & - & - & - & - & $\begin{array}{l}\text { Minimum, median, maxmum: } \\
\mathrm{SO}_{2} 0.33 \mathrm{ppb}, 2.1 \mathrm{ppb}, 51.4 \mathrm{ppb} \\
\mathrm{CO}, 0.15 \mathrm{ppm}, 0.9 \mathrm{ppm}, 13.5 \mathrm{ppm} ; \\
\mathrm{NO}_{2} 0.2 \mathrm{ppb}, 23.1 \mathrm{ppb}, 104.5 \mathrm{ppb} \\
\mathrm{O}_{3} 0.45 \mathrm{ppb}, 26.5 \mathrm{ppb}, 128 \mathrm{ppb} \\
\mathrm{PM}_{10}, 3.8 \mu \mathrm{g} / \mathrm{m}^{3}, 43,3183.4 \mu \mathrm{g} / \mathrm{m}^{3}\end{array}$ \\
\hline $\begin{array}{c}\text { Padula et al. } \\
2013 \text { [18] }\end{array}$ & - & - & - & - & $\begin{array}{c}\mathrm{CO}, \mathrm{Q} 1 \text { 0.13-0.39 ppm, Q4 0.72-1.37 ppm; } \\
\mathrm{NO}_{2} \text {, Q1 2.4-13.36 ppb, Q4 20.54-638.94 ppb; } \\
\mathrm{O}_{3} \text {, Q1 10.49-29.05 ppb, Q4 62.65-91.92 ppb; } \\
\mathrm{PM}_{10} \text {, Q1 7.9-25.24 } \mu \mathrm{g} / \mathrm{m}^{3}, \mathrm{Q} 4 \text { 44.09-95.32 } \mu \mathrm{g} / \mathrm{m}^{3}\end{array}$ \\
\hline $\begin{array}{l}\text { Dadvand et al. } \\
2011 \text { [29] }\end{array}$ & - & - & - & - & $\begin{array}{l}\text { Percentile 25- percentile 75: CO, 0.39-0.64 mg/m } 3 \text {; } \\
\qquad \mathrm{NO}_{2}, 29.2-38.4 \mu \mathrm{g} / \mathrm{m}^{3} ; \mathrm{NO}, 13.3-32.5 \mu \mathrm{g} / \mathrm{m}^{3} ; \\
\mathrm{O}_{3}, 33.2-42.4 \mu \mathrm{g} / \mathrm{m}^{3} ; \mathrm{PM}_{10}, 20.5-30.2 \mu \mathrm{g} / \mathrm{m}^{3}\end{array}$ \\
\hline $\begin{array}{l}\text { Dadvand } e t a l . \\
2011 \text { [28] }\end{array}$ & - & - & - & - & Percentile 25-percentile 75: $\mathrm{SO}_{2}, 17.6-31.2 \mu \mathrm{g} / \mathrm{m}^{3}$ \\
\hline $\begin{array}{l}\text { Marshall et al. } \\
2010 \text { [30] }\end{array}$ & & $\begin{array}{c}\text { Mean: } \\
\mathrm{PM}_{10}, 28.7 \mu \mathrm{g} / \mathrm{m}^{3} \\
\mathrm{NO}_{2}, 2.4 \mathrm{E}-2 \mathrm{ppm} \\
\mathrm{SO}_{2}, 5.3 \mathrm{E}-3 \mathrm{ppm} \\
\mathrm{O}_{3}, 2.5 \mathrm{E}-2 \mathrm{ppm} \\
\mathrm{CO}, 0.83 \mathrm{ppm}\end{array}$ & & $\begin{array}{c}\text { Mean: } \\
\mathrm{PM}_{10}, 28.1 \mu \mathrm{gg} \mathrm{m}^{3} \\
\mathrm{NO}_{2}, 2.4 \mathrm{E}-2 \mathrm{ppm} \\
\mathrm{SO}_{2}, 5.1 \mathrm{E}-3 \mathrm{ppm} \\
\mathrm{O}_{3}, 2.5 \mathrm{E}-2 \mathrm{ppm} \\
\mathrm{CO}, 0.85 \mathrm{ppm}\end{array}$ & \\
\hline
\end{tabular}


Table 2. Cont.

\begin{tabular}{|c|c|c|c|c|c|}
\hline \multirow{2}{*}{ Studies } & \multicolumn{2}{|c|}{ Case } & \multicolumn{2}{|c|}{ Control } & \multirow{2}{*}{ Total } \\
\hline & Congenital Defects & Air Pollutants & Congenital Defects & Air Pollutants & \\
\hline $\begin{array}{l}\text { Dolk et al. } \\
2010 *[31]\end{array}$ & - & - & - & - & $\begin{array}{c}\text { Percentile 10, median, percentile } 90: \\
\mathrm{SO}_{2}, 3.87 \mu \mathrm{g} / \mathrm{m}^{3}, 7.86 \mu \mathrm{g} / \mathrm{m}^{3}, 14.99 \mu \mathrm{g} / \mathrm{m}^{3} \\
\mathrm{NO}_{2}, 21.48 \mu \mathrm{g} / \mathrm{m}^{3}, 35.11 \mu \mathrm{g} / \mathrm{m}^{3}, 47.78 \mu \mathrm{g} / \mathrm{m}^{3} \\
\mathrm{PM}_{10}, 18.84 \mu \mathrm{g} / \mathrm{m}^{3}, 21.97 \mu \mathrm{g} / \mathrm{m}^{3}, 26.4 \mu \mathrm{g} / \mathrm{m}^{3}\end{array}$ \\
\hline $\begin{array}{l}\text { Hansen et al. } \\
2009[32]\end{array}$ & - & - & - & - & $\begin{array}{c}\text { Minmum, mean, maxmum: } \mathrm{SO}_{2}, 0,1.5 \mathrm{ppb}, 7.1 \mathrm{ppb} ; \\
\mathrm{CO}, 0.02 \mathrm{ppm}, 1.1 \mathrm{ppm}, 7.0 \mathrm{ppm} ; \\
\mathrm{NO}_{2}, 1.4 \mathrm{ppb}, 8.2 \mathrm{ppb}, 22.7 \mathrm{ppb} \\
\mathrm{O}_{3}, 4.3 \mathrm{ppb}, 25.8 \mathrm{ppb}, 54.4 \mathrm{ppb} \\
\mathrm{PM}_{10}, 4.4 \mu \mathrm{g} / \mathrm{m}^{3}, 18.0 \mu \mathrm{g} / \mathrm{m}^{3}, 151.7 \mu \mathrm{g} / \mathrm{m}^{3}\end{array}$ \\
\hline $\begin{array}{l}\text { Rankin et al. } \\
2009[33]\end{array}$ & - & $\begin{array}{l}\mathrm{SO}_{2} \mathrm{Q} 1-\mathrm{Q} 3 \\
2.7-4.4 \mu \mathrm{g} / \mathrm{m}^{3}\end{array}$ & - & - & 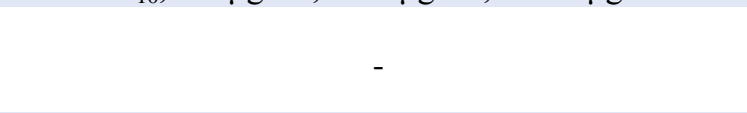 \\
\hline $\begin{array}{l}\text { Strickland et al. } \\
\qquad 2009 *[34]\end{array}$ & - & - & & - & $\begin{array}{c}\text { IQR: } \mathrm{SO}_{2}, 4.0 \mathrm{ppb} ; \mathrm{CO}, 0.3 \mathrm{ppm} ; \mathrm{NO}_{2}, 5.7 \mathrm{ppb} \\
\mathrm{O}_{3}, 29.9 \mu \mathrm{g} / \mathrm{m}^{3} ; \mathrm{PM}_{10}, 14.2 \mu \mathrm{g} / \mathrm{m}^{3}\end{array}$ \\
\hline $\begin{array}{c}\text { Hwang et al. } \\
2008[24]\end{array}$ & - & - & - & - & $\begin{array}{c}\text { Minmum, median, maxmum: } \\
\mathrm{O}_{3}, 16.7 \mathrm{ppb}, 26.8 \mathrm{ppb}, 45 \mathrm{ppb} \\
\mathrm{CO}, 25 \mathrm{pphm}, 62 \mathrm{pphm}, 277 \mathrm{pphm} \\
\mathrm{NO}_{\mathrm{x}}, 1.0 \mathrm{ppb}, 20.2 \mathrm{ppb}, 44.2 \mathrm{ppb} \\
\mathrm{PM}_{10}, 20.8 \mu \mathrm{g} / \mathrm{m}^{3}, 57.2 \mu \mathrm{g} / \mathrm{m}^{3}, 78.1 \mu \mathrm{g} / \mathrm{m}^{3}\end{array}$ \\
\hline $\begin{array}{c}\text { Gioboa et al. } \\
2005[36]\end{array}$ & - & - & - & - & $\begin{array}{c}\mathrm{CO}, \mathrm{Q} 1<0.4 \mathrm{ppm}, \mathrm{Q} 4 \geq 0.7 \mathrm{ppm} ; \\
\mathrm{NO}_{2}, \mathrm{Q} 1<1.3 \mathrm{pphm}, \mathrm{Q} 4 \geq 2.1 \mathrm{pphm} ; \\
\mathrm{O}_{3}, \mathrm{Q} 1<1.8 \mathrm{pphm}, \mathrm{Q} 4 \geq 3.1 \mathrm{pphm} ; \\
\mathrm{SO}_{2}, \mathrm{Q} 1<1.3 \mathrm{ppb}, \mathrm{Q} 4 \geq 2.7 \mathrm{ppb} ; \\
\mathrm{PM}_{10}, \mathrm{Q} 1<19.5 \mu \mathrm{g} / \mathrm{m}^{3}, \mathrm{Q} 4 \geq 29 \mu \mathrm{g} / \mathrm{m}^{3}\end{array}$ \\
\hline
\end{tabular}

Notes: CHD, congenital heart defects; VSD, ventricular heart defects; IQR, interquartile range.; Q1, quartile 1; Q2, quartile 2; Q3, quartile 3; Q4, quartile 4;

* indicated cohort studies, others were designed as case-control studies; “"” no information. 
Regarding cardiovascular anomalies, 16 combinations of pollutants-cardiac anomalies could be included in the meta-analysis (Figures 1-3). In all, exposure was expressed as continuous variables. We found a significantly increased meta-OR for exposure to $\mathrm{NO}_{2}$ and the risk of coarctation of aorta (OR per $10 \mathrm{ppb}=1.20,95 \% \mathrm{CI}(1.02,1.41)$ ), which is in accord with the previous meta-analysis [16]. For all other combinations, the combined effects were close to one and not significant.

Figure 1. Forest plots for combinations of ventricular septal defects and pollutant (as a continuous variable). The size of each square represents the weight that contributes to the combined effect, respectively for: $(\mathbf{A}) \mathrm{SO}_{2} ;(\mathbf{B}) \mathrm{PM}_{10} ;(\mathbf{C}) \mathrm{NO}_{2} ;(\mathbf{D}) \mathrm{CO}$; and (E) $\mathrm{O}_{3}$.

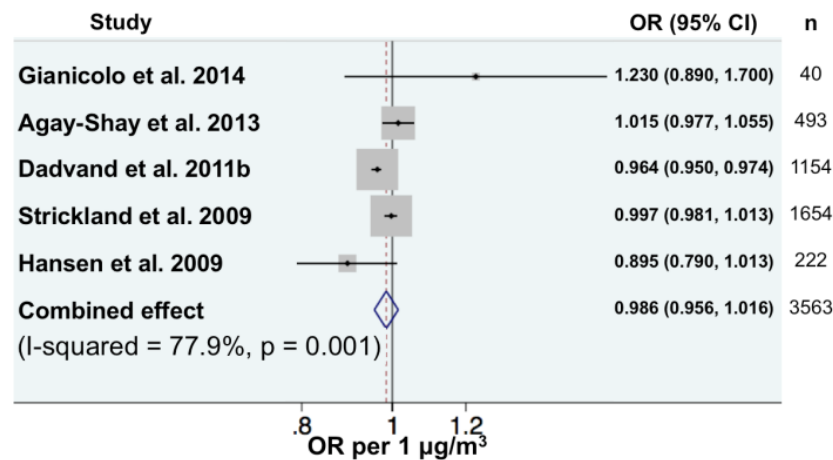

(A) $\mathrm{SO}_{2}$ and ventricular septal defects Study

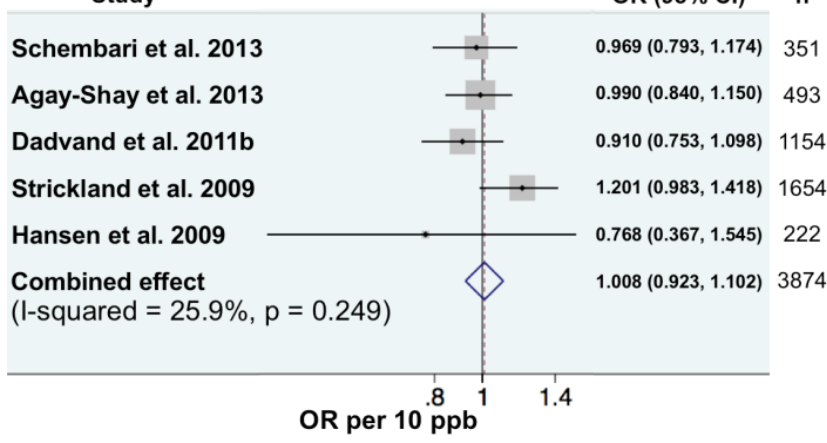

(C) $\mathrm{NO}_{2}$ and ventricular septal defects
Study

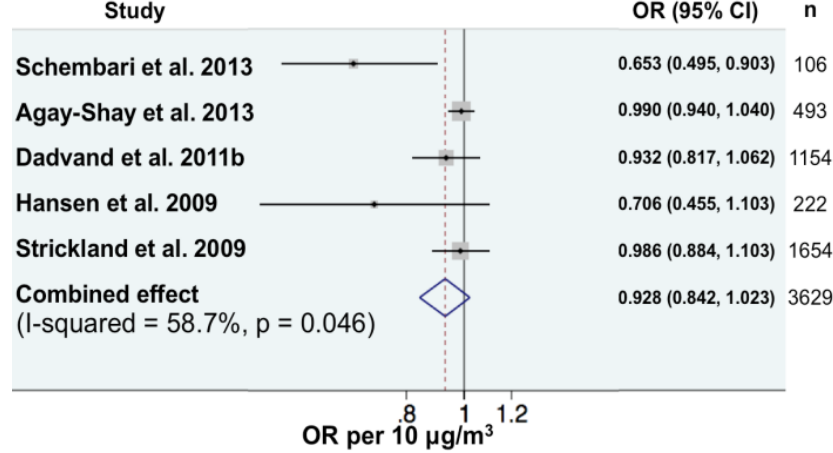

(B) $\mathrm{PM}_{10}$ and ventricular septal defects Study

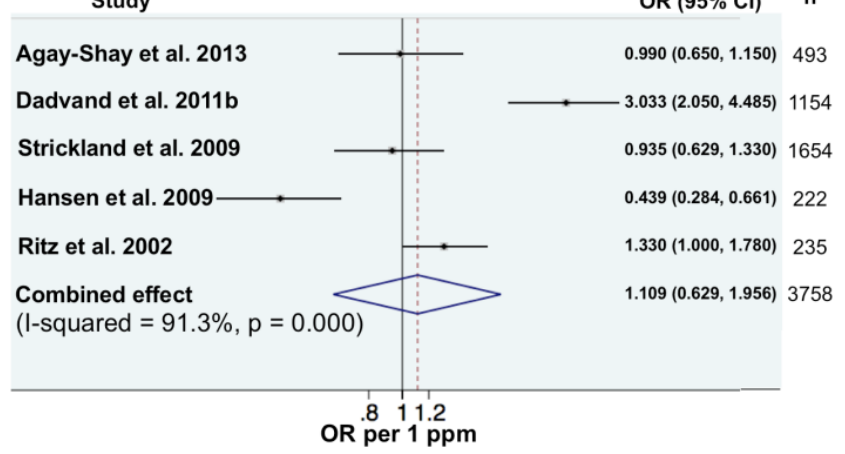

(D) $\mathrm{CO}$ and ventricular septal defects OR $(95 \% \mathrm{Cl}) \quad n$

$0.950(0.870,1.040) 493$

$1.041(0.951,1.149) 1154$

$1.011(0.967,1.059) 1654$

$0.820(0.660,1.010) 222$

$1.842(0.418,8.114) 261$

$0.999(0.963,1.036) 3784$

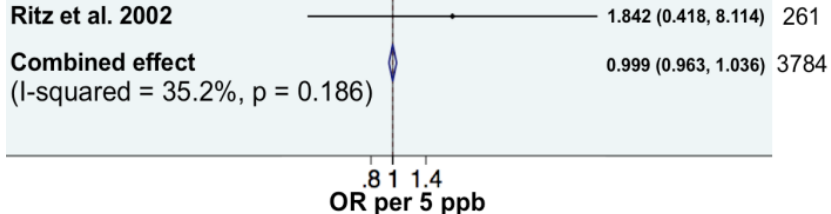

(E) $\mathrm{O}_{3}$ and ventricular septal defects 
Figure 2. Forest plots for combinations of atrial septal defects and pollutant (as a continuous variable). The size of each square represents the weight that contributes to the combined effect, respectively for: (A) $\mathrm{SO}_{2} ;(\mathbf{B}) \mathrm{PM}_{10} ;(\mathbf{C}) \mathrm{NO}_{2} ;$ (D) $\mathrm{CO}$ and (E) $\mathrm{O}_{3}$.

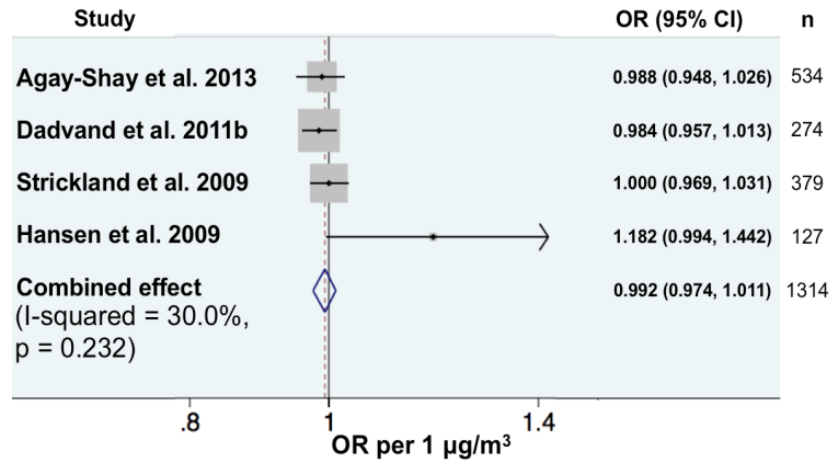

(A) $\mathrm{SO}_{2}$ and atrial septal defects Study

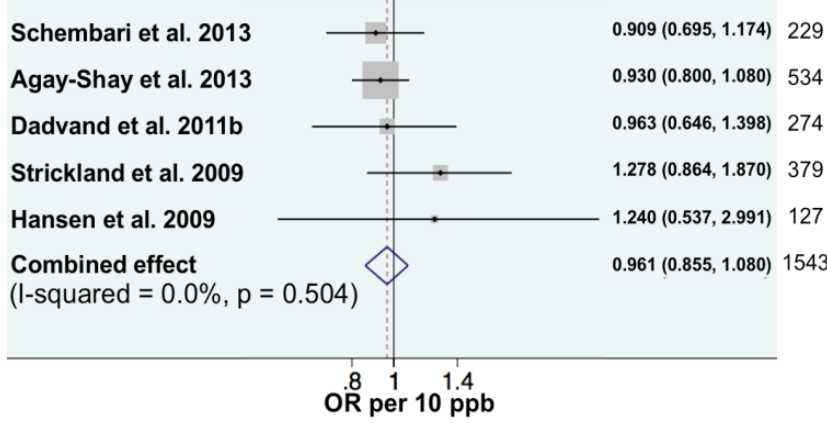

(C) $\mathrm{NO}_{2}$ and atrial septal defects

Study

Agay-Shay et al. 2013

Dadvand et al. 2011b

Strickland et al. 2009

Hansen et al. 2009

Ritz et al. 2002

Combined effect

(I-squared $=0.0 \%, p=0.706)$
Study

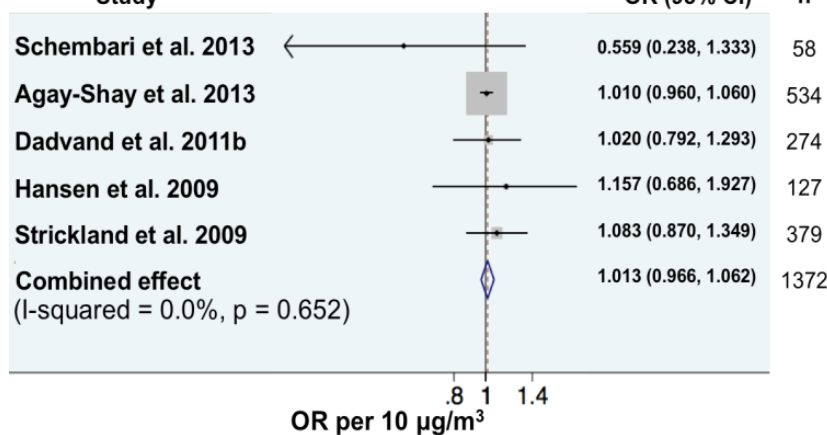

(B) $\mathrm{PM}_{10}$ and atrial septal defects Study

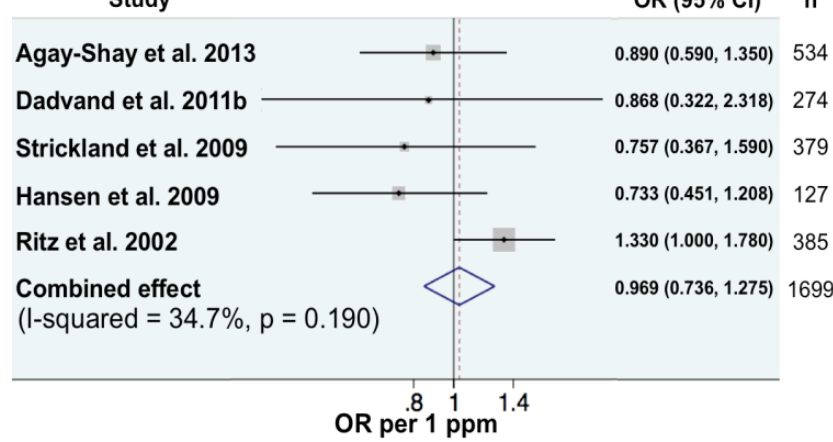

(D) CO and atrial septal defects OR $(95 \% \mathrm{Cl}) \quad \mathrm{n}$

$1.010(0.920,1.110) 534$

$1.062(0.868,1.280) \quad 274$

$1.025(0.935,1.123) \quad 379$

$1.030(0.770,1.370) \quad 127$

$0.444(0.135,1.403) \quad 385$

$1.020(0.960,1.084) 1699$

OR per 5 ppb

(E) $\mathrm{O}_{3}$ and atrial septal defects 
Figure 3. Forest plots for combinations of two cardiac anomalies (coarctation of aorta and tetralogy of fallot) and pollutant. The size of each square represents the weight that contributes to the combined effect. $(\mathbf{A}),(\mathbf{C}),(\mathbf{E})$ were combined effects of coarctation of aorta and $\mathrm{SO}_{2}, \mathrm{PM}_{10}$ and $\mathrm{NO}_{2}$. (B), (D) and (F) were combined effects of tetralogy of fallot and $\mathrm{SO}_{2}, \mathrm{PM}_{10}$ and $\mathrm{NO}_{2}$.

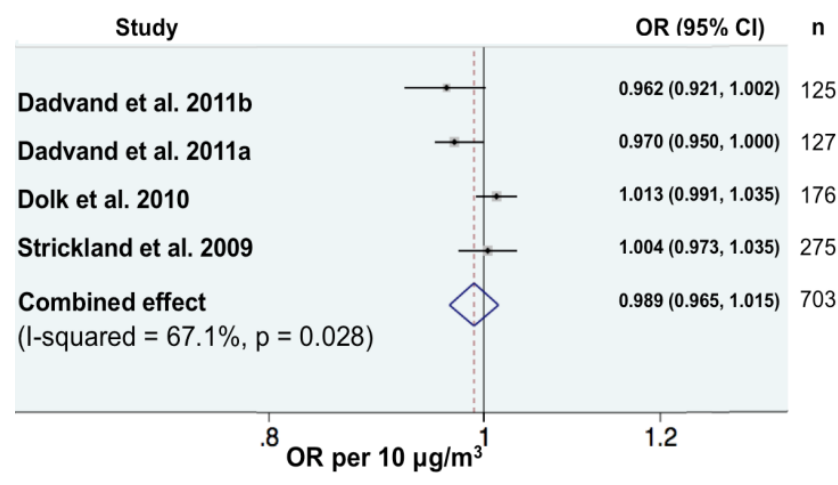
tudy

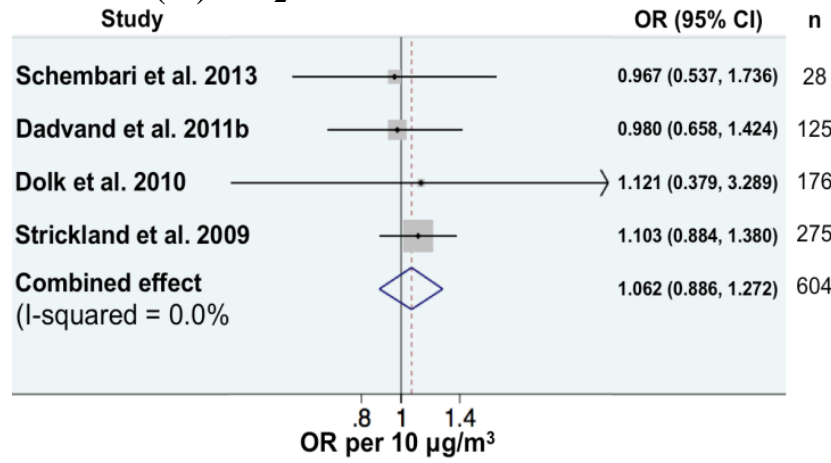

(C) $\mathrm{PM}_{10}$ and coarctation of aorta Study

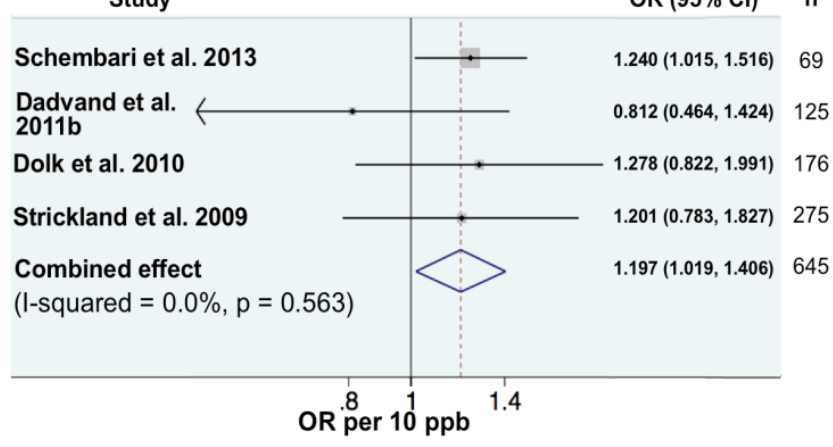

(E) $\mathrm{NO}_{2}$ and coarctation of aorta

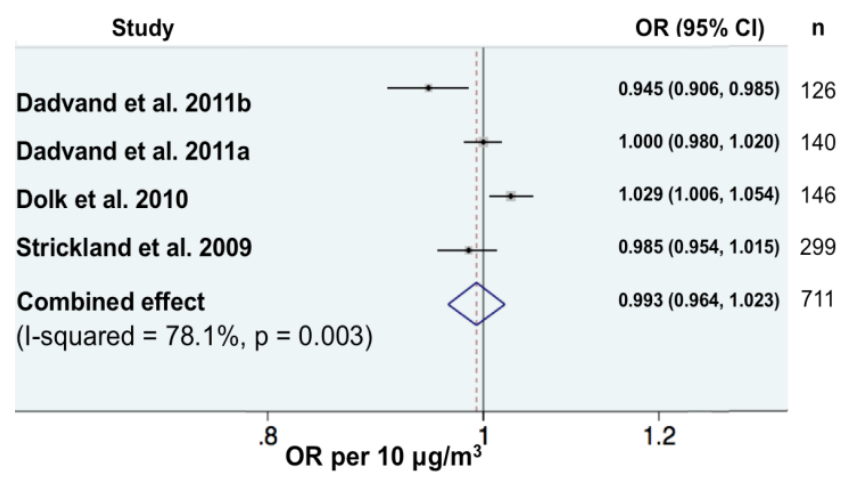

(B) $\mathrm{SO}_{2}$ and tetralogy of fallot

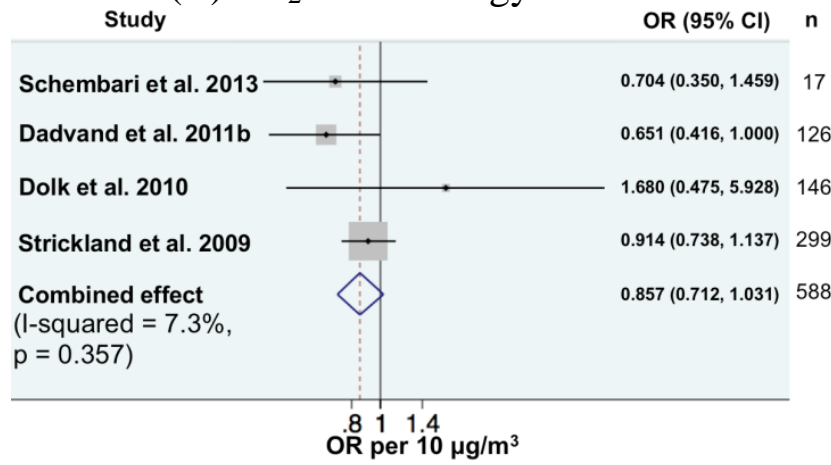

(D) $\mathrm{PM}_{10}$ and tetralogy of fallot

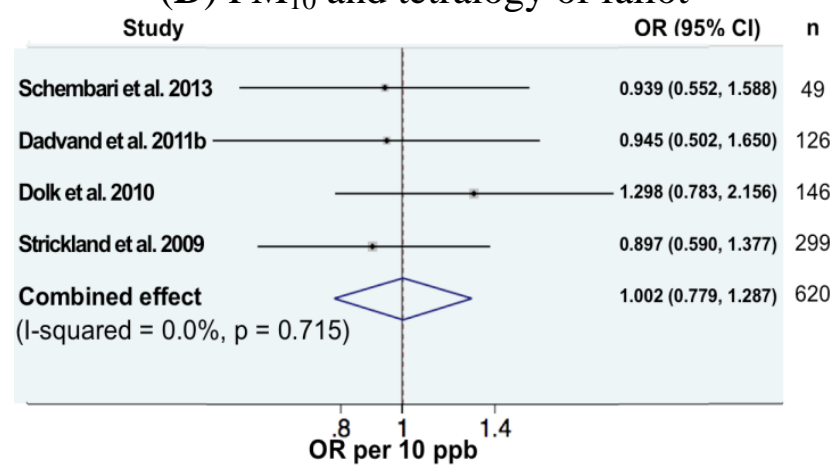

(F) $\mathrm{NO}_{2}$ and tetralogy of fallot

For oro-facial clefts, seven articles were included in the meta-analysis, with one study exhibiting a statistically significant association [24]: the OR comparing the 4th with the 1st quartiles of the exposure distribution was equal to 1.40 (95\% CI $(1.05,1.91))$. The meta-analysis found no significant association for the four other pollutants (Figure 4A-D). In most cases, the studies on cleft lips, for which exposure was expressed on a continuous scale, were less than four, except for those exploring the effect of exposure to $\mathrm{NO}_{2}$ whose result is presented in Figure 4E also showing no significant association. 
Figure 4. Forest plots for a variety of pollutants and risk of orofacial defects. (A), (B), (C) and (D) showed combined effects of $\mathrm{PM}_{10}, \mathrm{NO}_{2}, \mathrm{CO}$ and $\mathrm{O}_{3}$; (E) was the combined effect of $\mathrm{NO}_{2}$ and cleft lip from continuous exposure risk estimates.

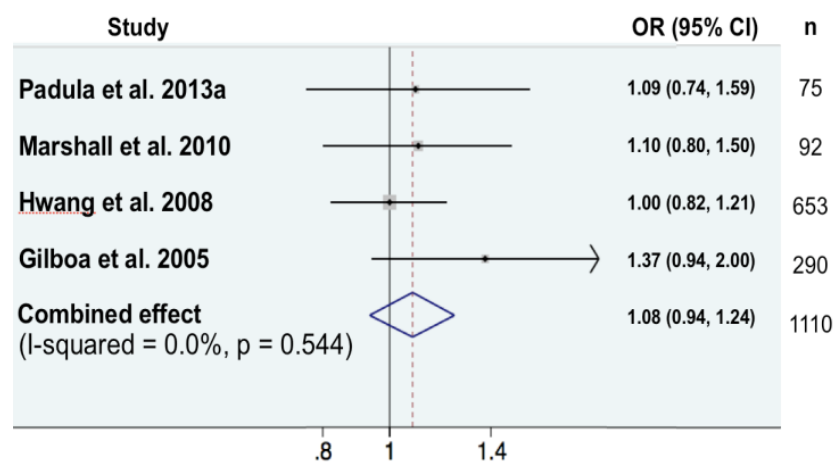

(A) $\mathrm{PM}_{10}$ and cleft lip

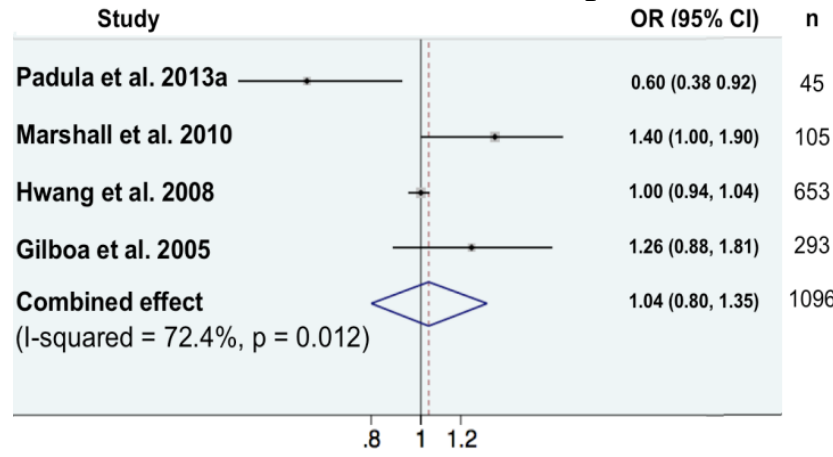

(C) $\mathrm{CO}$ and cleft lip $\operatorname{lip}_{\text {Study }}$

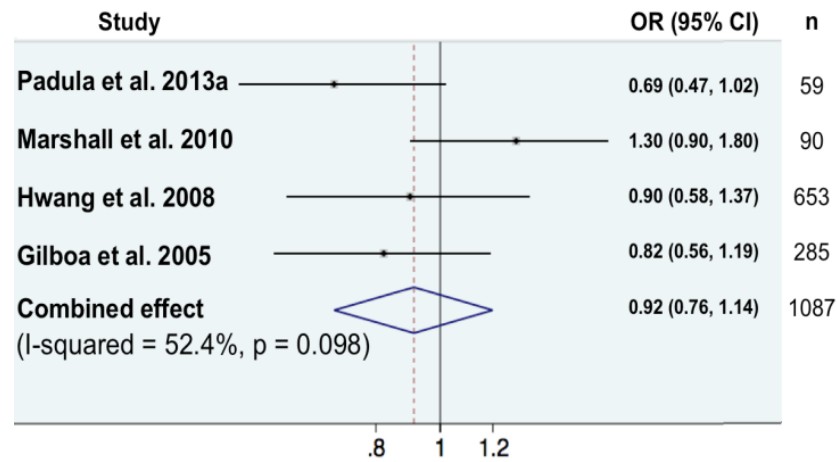

(B) $\mathrm{NO}_{2}$ and cleft lip

Study

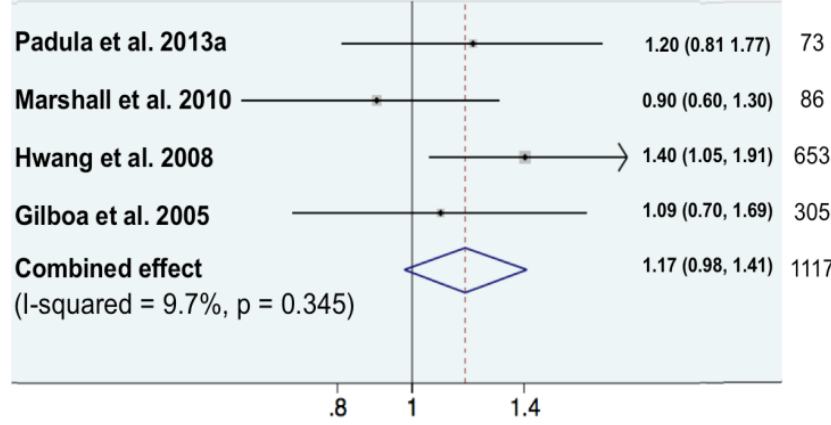

(D) $\mathrm{O}_{3}$ and cleft lip

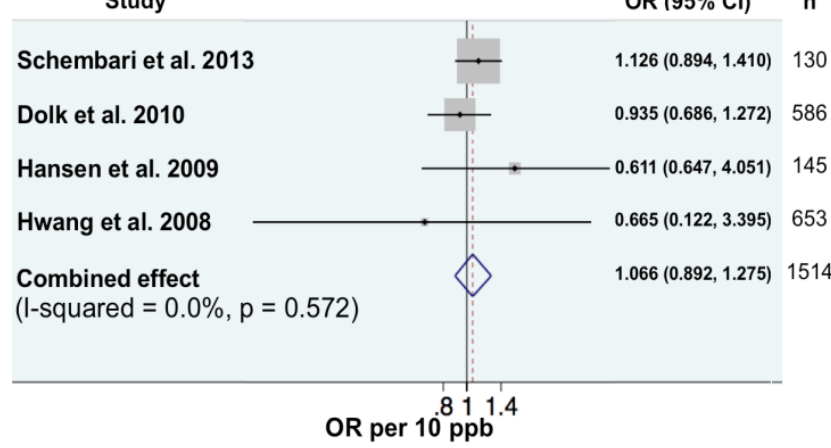

(E) $\mathrm{NO}_{2}$ and cleft lip

\section{Discussion}

Congenital anomalies are the leading cause of neonatal mortality. The prevalence in France reported by the national health surveillance institute is equal to 3.3\% [37]. In this systematic review, we assessed the effects of air pollution on the risk of congenital anomalies based on the epidemiologic literature. We compared 21 combinations of air pollutants and congenital anomalies types and only one significant result was revealed. The input data of the 21 combinations were different; it is improbable that the number of statistical tests performed explains the result. But, as all significant results, we cannot exclude that it is a chance finding. The combined risk of coarctation of aorta was found significantly associated with $\mathrm{NO}_{2}(\mathrm{OR}$ per $10 \mathrm{ppb}=1.20,95 \% \mathrm{CI}(1.02,1.41))$. Our meta-analysis did 
not reveal any other significant association for cardiac anomalies and oral-facial defects, which is in coherence with the previous meta-analysis published in 2010 by Vrijheid et al. [16].

However, these authors revealed a significantly increased risk between exposure to $\mathrm{NO}_{2}, \mathrm{SO}_{2}$ and the tetralogy of fallot (OR per $10 \mathrm{ppb} \mathrm{NO}=1.25,95 \% \mathrm{CI}(1.02-1.51)$; and OR per 1 ppb $\mathrm{SO}_{2}=1.04$, 95\% CI (1.00-1.08), respectively) and coarctation of aorta (OR per $10 \mathrm{ppb} \mathrm{NO}_{2}=1.20,95 \% \mathrm{CI}$ (1.00-1.44); OR per 1 ppb $\mathrm{SO}_{2}=1.04$; 95\% CI (1.00-1.08)). We did not undertake the meta-analysis for these combinations because the number of studies was small. In the present work, we chose to realize two-separate meta-analyses according to the type (continuous or categorical) of the exposure variable, not to introduce too much heterogeneity in the meta-estimates, whereas Vrijheid et al. converted all continuous to categorical variables in order to increase their statistical power.

The present meta-analysis has several limitations. The first one deals with the sample size. Most of the combined effects were computed with about five studies; we did not try to compute combined effects for less than four studies. There was, however, a large total number of cases included in the meta-analysis (varying from 588 to 3874 according to the air pollutant-congenital anomaly combination), that provided enough statistical power to reveal significant associations. The numbers of cases and controls of each study are reported in Tables 3-7. We calculated the statistical power (a fixed alpha-risk $=5 \%$ ) for the smallest sample size (Table 7) and the highest (Tables 5 and 6). With a statistical power equal to $90 \%$, we will detect a statically significant health effect equal to $18 \%$ and $25 \%$ from the smallest and the highest sample size, respectively; note that, due to the small number of cases counted in the highest sample size (about 600 cases among a population of about 1.5 million because of the cohort studies include in the meta analysis), a smaller effect could be found significant from the smallest sample size. The statistical power will fall to $70 \%$ and $60 \%$ (respectively, in the smallest and highest samples sizes) to reveal a significant increase of the risk equal to $10 \%$.

Secondly, we found differences in the methodologies adopted in the 17 individual studies included in the present review, which make difficult the comparisons and assessment of the combined effects. Certain studies relied on measurement of air quality at the birth residence $[9,28-33,36]$, which is not necessarily the address during early pregnancy (the critical window time) and hence may lead to misclassification of exposure for several pregnant women. In addition, without distance-weighted calculation in the exposure assessments, the range of distance between maternal place and the nearest monitoring station may produce uncertain exposure estimates and thus lead also to exposure misclassification. 
Table 3. Numbers of ventricular septal defects cases and number of controls in studies included in the meta analysis for 5 pollutants: $\mathrm{SO}_{2}, \mathrm{PM}_{10}, \mathrm{NO}_{2}, \mathrm{CO}, \mathrm{O}_{3}$.

\begin{tabular}{|c|c|c|c|c|c|c|c|c|c|c|c|c|c|c|c|c|}
\hline \multirow[t]{2}{*}{$\begin{array}{l}\text { Numbers of cases and controls exposed under various } \\
\text { pollutants among different studies }\end{array}$} & \multicolumn{2}{|c|}{$\begin{array}{l}\text { Gianicolo et al. } \\
2014[23]\end{array}$} & \multicolumn{2}{|c|}{$\begin{array}{c}\text { Schembari } \\
\text { et al. } 2013 \\
{[22]}\end{array}$} & \multicolumn{2}{|c|}{$\begin{array}{c}\text { Agay-Shay } \\
\text { et al. } 2013 \\
{[21]} \\
\end{array}$} & \multicolumn{2}{|c|}{$\begin{array}{c}\text { Dadvand } \\
\text { et al. } 2011 \\
{[29]} \\
\end{array}$} & \multicolumn{2}{|c|}{$\begin{array}{l}\text { Strickland et al. } \\
\quad 2009[34]\end{array}$} & \multicolumn{2}{|c|}{$\begin{array}{l}\text { Hansen et al. } \\
2009[32]\end{array}$} & \multicolumn{2}{|c|}{$\begin{array}{c}\text { Ritz et al. } \\
2002[9]\end{array}$} & \multicolumn{2}{|c|}{ Total Number } \\
\hline & case & control & case & control & case & control & case & control & case & control & case & control & case & control & case & control \\
\hline $\mathrm{SO}_{2}$ & 40 & 150 & - & - & 493 & 130,402 & 1154 & 4616 & 1654 & 713,846 & 222 & 1110 & 235 & 9049 & 3798 & 859,173 \\
\hline $\mathrm{PM}_{10}$ & - & - & 106 & 903 & 493 & 130,402 & 1154 & 4616 & 1654 & 713,846 & 222 & 1110 & 235 & 9049 & 3864 & 859,926 \\
\hline $\mathrm{NO}_{2}$ & - & - & 351 & 2869 & 493 & 130,402 & 1154 & 4616 & 1654 & 713,846 & 222 & 1110 & - & - & 3874 & 852,843 \\
\hline $\mathrm{CO}$ & - & - & - & - & 493 & 130,402 & 1154 & 4616 & 1654 & 713,846 & 222 & 1110 & - & - & 3523 & 849,974 \\
\hline $\mathrm{O}_{3}$ & - & - & - & - & 493 & 130,402 & 1154 & 4616 & 1654 & 713,846 & 222 & 1110 & - & - & 3523 & 849,974 \\
\hline
\end{tabular}

Table 4. Numbers of atrial septal defects cases and number of controls in studies included in the meta analysis for 5 pollutants: $\mathrm{SO}_{2}$, $\mathrm{PM}_{10}$, $\mathrm{NO}_{2}, \mathrm{CO}, \mathrm{O}_{3}$.

\begin{tabular}{|c|c|c|c|c|c|c|c|c|c|c|c|c|c|c|}
\hline \multirow[t]{2}{*}{$\begin{array}{c}\text { Numbers of cases and controls exposed under various } \\
\text { pollutants among different studies }\end{array}$} & \multicolumn{2}{|c|}{$\begin{array}{c}\text { Schembari et al. } \\
2013[22] \\
\end{array}$} & \multicolumn{2}{|c|}{$\begin{array}{c}\text { Agay-Shay et al. } \\
2013[21] \\
\end{array}$} & \multicolumn{2}{|c|}{$\begin{array}{c}\text { Dadvand et al. } \\
2011[29]\end{array}$} & \multicolumn{2}{|c|}{$\begin{array}{c}\text { Strickland et al. } \\
2009[34]\end{array}$} & \multicolumn{2}{|c|}{$\begin{array}{c}\text { Hansen et al. } \\
2009[32]\end{array}$} & \multicolumn{2}{|c|}{$\begin{array}{c}\text { Ritz et al. } \\
2002[9]\end{array}$} & \multicolumn{2}{|c|}{ Total Number } \\
\hline & case & control & case & control & case & control & case & control & case & control & case & control & case & control \\
\hline $\mathrm{SO}_{2}$ & - & - & 534 & 130,402 & 274 & 1096 & 379 & 715,121 & 127 & 635 & - & - & 1314 & 847,254 \\
\hline $\mathrm{PM}_{10}$ & 106 & 903 & 534 & 130,402 & 274 & 1096 & 379 & 715,121 & 127 & 635 & - & - & 1420 & 848,157 \\
\hline $\mathrm{NO}_{2}$ & 229 & 2869 & 534 & 130,402 & 274 & 1096 & 379 & 715,121 & 127 & 635 & - & - & 1543 & 850,123 \\
\hline $\mathrm{CO}$ & - & - & 534 & 130,402 & 274 & 1096 & 379 & 715,121 & 127 & 635 & 385 & 3000 & 1699 & 850,254 \\
\hline $\mathrm{O}_{3}$ & - & - & 534 & 130,402 & 274 & 1096 & 379 & 715,121 & 127 & 635 & 385 & 3000 & 1699 & 850,254 \\
\hline
\end{tabular}


Table 5. Numbers of coarctation of aorta cases and number of controls in studies included in the meta analysis for 3 pollutants: $\mathrm{SO}_{2}, \mathrm{PM}_{10}, \mathrm{NO}_{2}$.

\begin{tabular}{|c|c|c|c|c|c|c|c|c|c|c|c|c|}
\hline \multirow[t]{2}{*}{$\begin{array}{c}\text { Numbers of cases and controls exposed under various } \\
\text { pollutants among different studies }\end{array}$} & \multicolumn{2}{|c|}{$\begin{array}{c}\text { Schembari } \text { et al. } \\
\quad 2013 \text { [22] }\end{array}$} & \multicolumn{2}{|c|}{$\begin{array}{c}\text { Dadvand et al. } \\
\quad 2011[29]\end{array}$} & \multicolumn{2}{|c|}{$\begin{array}{c}\text { Dadvand } \text { et al. } \\
\quad 2011[28]\end{array}$} & \multicolumn{2}{|c|}{$\begin{array}{l}\text { Dolk et al. } \\
2010[31]\end{array}$} & \multicolumn{2}{|c|}{$\begin{array}{c}\text { Strickland et al. } 2009 \\
{[34]}\end{array}$} & \multicolumn{2}{|c|}{ Total Number } \\
\hline & case & control & case & control & case & control & case & control & case & control & case & control \\
\hline $\mathrm{SO}_{2}$ & - & - & 125 & 500 & 127 & 508 & 176 & 759,817 & 275 & 715225 & 703 & $1,476,050$ \\
\hline $\mathrm{PM}_{10}$ & 28 & 890 & 125 & 500 & - & - & 176 & 759,817 & 275 & 715225 & 604 & $1,476,432$ \\
\hline $\mathrm{NO}_{2}$ & 69 & 2869 & 125 & 500 & - & - & 176 & 759,817 & 127 & 715225 & 497 & $1,478,411$ \\
\hline
\end{tabular}

Table 6. Numbers of tetralogy of fallot cases and number of controls in studies included in the meta analysis for the 3 pollutants: $\mathrm{SO}_{2}, \mathrm{PM}_{10}, \mathrm{NO}_{2}$.

\begin{tabular}{|c|c|c|c|c|c|c|c|c|c|c|c|c|}
\hline \multirow[t]{2}{*}{$\begin{array}{c}\text { Numbers of cases and controls exposed under various } \\
\text { pollutants among different studies } \\
\end{array}$} & \multicolumn{2}{|c|}{$\begin{array}{l}\text { Schembari et al. } \\
2013[22]\end{array}$} & \multicolumn{2}{|c|}{$\begin{array}{l}\text { Dadvand et al. } \\
2011[29] \\
\end{array}$} & \multicolumn{2}{|c|}{$\begin{array}{l}\text { Dadvand et al. } \\
2011[28] \\
\end{array}$} & \multicolumn{2}{|c|}{$\begin{array}{l}\text { Dolk et al. } \\
2010[31]\end{array}$} & \multicolumn{2}{|c|}{$\begin{array}{c}\text { Strickland } \text { et al. } \\
2009[34]\end{array}$} & \multicolumn{2}{|c|}{ Total Number } \\
\hline & case & control & case & control & case & control & case & control & case & control & case & control \\
\hline $\mathrm{SO}_{2}$ & - & - & 126 & 504 & 140 & 560 & 146 & 759,817 & 299 & 715,201 & 711 & $1,476,082$ \\
\hline $\mathrm{PM}_{10}$ & 17 & 890 & 126 & 504 & - & - & 146 & 759,817 & 299 & 715,201 & 588 & $1,476,412$ \\
\hline $\mathrm{NO}_{2}$ & 49 & 2650 & 126 & 504 & - & - & 146 & 759,817 & 299 & 715,201 & 620 & $1,478,172$ \\
\hline
\end{tabular}

Table 7. Numbers of cleft lip cases and number of controls in studies included in the meta analysis for 4 pollutants: $\mathrm{PM}_{10}, \mathrm{NO}_{2}, \mathrm{CO}, \mathrm{O}_{3}$.

\begin{tabular}{|c|c|c|c|c|c|c|c|c|c|c|}
\hline \multirow{2}{*}{$\begin{array}{l}\text { Numbers of cases and controls exposed under } \\
\text { various pollutants among different studies }\end{array}$} & \multicolumn{2}{|c|}{ Padula et al. 2013 [18] } & \multicolumn{2}{|c|}{ Marshall et al. 2010 [30] } & \multicolumn{2}{|c|}{ Hwang et al. 2008 [24] } & \multicolumn{2}{|c|}{ Giloba et al. 2005 [36] } & \multicolumn{2}{|c|}{ Total Number } \\
\hline & case & control & case & control & case & control & case & control & case & control \\
\hline $\mathrm{PM}_{10}$ & 75 & 200 & 92 & 12,925 & 653 & 6530 & 290 & 3450 & 1110 & 23,105 \\
\hline $\mathrm{NO}_{2}$ & 59 & 205 & 92 & 12,925 & 653 & 6530 & 285 & 3237 & 1089 & 22,897 \\
\hline $\mathrm{CO}$ & 45 & 157 & 92 & 12,925 & 653 & 6530 & 293 & 3309 & 1083 & 22,921 \\
\hline $\mathrm{O}_{3}$ & 73 & 201 & 92 & 12,925 & 653 & 6530 & 305 & 3594 & 1123 & 23,250 \\
\hline
\end{tabular}


Different classifications of congenital anomalies also yield some heterogeneity. Specific definition of defects could ease detection of significant associations. Ventricular septal defects are the most common congenital subgroups that were studied and a variety of classifications were found. For example, ventricular septal defects were classified into three subgroups (ventricular septal defects-permembranous, muscular and conov) in the Padula et al. study [19], according to the classification proposed by Botto et al. [38], whereas in others they used two subgroups (ventricular septal defect, muscular and permembranous) [31] or only one global group [21,29,32,36]. Another example is the subcategory "conotruncal defect": four studies used this outcome category to include other cardiovascular malformations, such as "tetralogy of fallot", "transposition of the great arteries", truncus arteriosus communis, "double outlet right ventricle" or "aorticopulmonary window" [9,31,32,36]; while, tetralogy of fallot and transposition of the great arteries were considered as an event in most other studies.

Confounding factors included in the individual studies are an additional problem. Few confounders, such as smoking [39], parental occupation [40], maternal age [41] and season conception [42] have been addressed in congenital anomalies research. Season and maternal age at conception were the most frequent confounders considered in the studies included in the present work. Seasonal variations of congenital anomalies incidence have been well described, with a higher risk in summer than in winter [42]. The association between maternal age and the risk of non-genetic congenital anomalies is still unclear. Previous studies analyzing the EUROCAT database found that teenage mothers were at higher risk but not older mothers (35-44 years). However, among European countries, the maternal age patterns are less clear. This suggests the influence of social factors, exposures or living habits, which might be associated with maternal age [41]. The prevalence of congenital anomalies is related to the social status, with higher values in deprived categories [43]. Now, few studies have addressed socioeconomic status/deprivation as confounders or effect modifiers [22,27,28,36]. One study matched cases and controls according to a neighborhood socioeconomic deprivation index but without considering other confounders in the statistical analysis [23]. One may consider that socioeconomic deprivation would constitute a good approximation of well-known risk factors of congenital abnormalities such as smoking habits or educational level. The wide range of confounders considered in the individual studies included in the present review might introduce heterogeneity when combining the data. Because of these limitations, this meta-analysis could detect only few significant associations between air pollution and birth defects; however it does not mean that the hypothesis should be definitively disregarded.

The different methods used in the studies, such as case and control definition, exposure assessment and confounding factors as all mentioned earlier, could damage the quality level of each study included in the meta-analysis and consequently the quality of the combined estimates. Assessing the quality of studies is important to understand properly each study to be used in meta analysis. It would be interesting to use the Newcastle-Ottawa Scale (NOS) [44] followed by quality score analysis as recommended by Detsky et al. [45] in order to assess the quality of each study. Then, including only studies with the highest quality score in the meta-analyses, we could measure more precisely the impact of the study quality on the point estimates. However, due to the limited number of studies in our meta-analyses, it was not possible to conduct this procedure. 
Language selection may also bias the data basis. Non-English publications of relevant articles may have been ignored. Moreover, the risk of publication bias is inherent in systematic literature review. Unpublished results (probably, more likely to bear not significant results and the grey literature, which is not available on open sources) may distort the meta-analysis findings. So far, the two meta-analyses (the one published in 2010 and the present one) tend to suggest an adverse effect of air pollution on at least one type of birth defects, and this call for further studies in order to confirm the finding. We failed to assess publication bias by using funnel plots. According to the recommendations from Cochrane Handbook for Systematic Reviews of Interventions [46], there should be at least 10 studies in the meta-analysis to distinguish real asymmetry.

Air pollutants could directly exert adverse effects as pro-oxidants binding to lipid and proteins, therefore promoting oxidative stress and the production of free radicals, a process that may elicit a variety of diseases or defects [47]. This oxidative stress caused by air pollution during pregnancy has been pointed out in some studies [48,49]. In addition, there is recent evidence that air pollutants can contribute to epigenetic changes, including alteration of DNA methylation [50]. MicroRNA has been also studied with regard of the environmental changes and there is evidence that microRNA expression and regulation may be affected by environmental exposures, such as air pollution, smoking and heavy metal accumulation [51]. Such epigenetic modifications during pregnancy could impair normal embryo development and lead to birth defects.

\section{Conclusions}

Air pollution is a universal issue. Therefore, a small increase in risks may lead to serious public health problems. Congenital anomalies are the main causes of preterm and neonatal mortality and morbidity. Meta-analysis is an appropriate tool to enhance statistical power in the analysis of weak associations. It might shed new light on the association between air pollution and congenital anomalies insofar as new studies are conducted that overcome the limitations discussed in the present literature review. Improved exposure assessment methods, in particular more accurate spatial measurements or modeling, standardized definition of cases and accommodation of known or putative confounders are highly recommended for future congenital anomalies research on the effect of air pollution.

\section{Acknowledgments}

This work is supported by Fondation de France and the EHESP School of Public Health.

\section{Author Contributions}

Esther Chen has conducted the statistical analysis and written the article under the supervision of Séverine Deguen. Séverine Deguen, coordinator of research projects dealing with environmental exposure related to social health inequalities including the congenital malformation, insured the good realization of the statistical analysis and their interpretation; she contributed also to the written of the article. Cindy Padilla and Denis Zmirou re-read the article and contributed to its improvement. 


\section{Conflicts of Interest}

The authors declare no conflict of interest.

\section{References}

1. McConnell, R.; Islam, T.; Shankardass, K.; Jerrett, M.; Lurmann, F.; Gilliland, F.; Gauderman, J.; Avol, E.; Künzli, N.; Yao, L.; et al. Childhood incident asthma and traffic-related air pollution at home and school. Environ. Health Perspect. 2010, 118, 1021-1026.

2. Zmirou, D.; Gauvin, S.; Pin, I.; Momas, I.; Sahraoui, F.; Just, J.; le Moullec, Y.; Brémont, F.; Cassadou, S.; Reungoat, P.; et al. Traffic-related air pollution and incidence of childhood asthma: Results of the VESTA case control study. J. Epidemiol. Community Health 2004, 58, 18-23.

3. Ko, F.W.; Hui, D.S. Air pollution and chronic obstructive pulmonary disease. Respirology 2012, 17, 395-401.

4. Pope, C.A., III; Burnett, R.T.; Thun, M.J.; Calle, E.E.; Ito, K.; Thurston, G.D. Lung cancer, cardiopulmonary mortality, and long-term exposure to fine particulate air pollution. JAMA 2002, 287, 1132-1141.

5. Nafstad, P.; Haheim, L.L.; Ofterdal, B.; Gram, F.; Holme, I.; Hjermann, I.; Leren, P. Lung cancer and air pollution: A 27 year follow up of 16,209 Norwegian men. Thorax 2003, 58, 1071-1076.

6. Brunekreef, B.; Beelen, R.; Hoek, G.; Schouten, L.; Bausch-Goldbohm, S.; Fischer, P.; et al. Effects of long-term exposure to traffic-related air pollution on respiratory and cardiovascular mortality in the Netherlands: the NLCS-AIR study. Res. Rep. Health Eff. Inst. 2009, 139, 5-71.

7. Nieuwenhuijsen, M.J.; Basagaña, X.; Dadvand, P.; Martinez, D.; Cirach, M.; Beelen, R.; Jacquemin, B. Air pollution and human fertility rates. Environ. Int. 2014, 27, 9-14.

8. Air Quality Guidelines; WHO: Copenhagen, Denmark, 2005.

9. Ritz, B.; Yu, F.; Fruin, S.; Chapa, G.; Shaw, G.M.; Harris, J.A. Ambient air pollution and risk of birth defects in southern California. Amer. J. Epidemiol. 2002, 155, 17-25.

10. Karr, C.; Lumley, T.; Schreuder, A.; Davis, R.; Larson, T.; Ritz, B. Effects of subchronic and chronic exposure to ambient air pollutants on infant bronchiolitis. Amer. J. Epidemiol. 2007, 165, 553-560.

11. Pedersen, M.; Giorgis-Allemand, L.; Bernard, C.; Aguilera, I.; Anderson, A.M.; Ballester, F.; Beelen, R.M.; Chatzi, L.; Cirach, M.; Danileviciute, A.; et al. Ambient air pollution and low birthweight: A European cohort study (ESCAPE). Lancet Respir. Med. 2013, 1, 695-704.

12. Effects of Air Pollution on Children's Health and Development; WHO: Copenhagen, Denmark, 2005.

13. World Health Statistics; WHO: Geneva, Switzerland, 2013.

14. Perinatal Mortality Associated with Congenital Anomalies in EUROCAT Full Member Registries, 2008-2012; European Surveillance of Congenital Anomalies (EUROCAT). Table 1. Available online: http://www.eurocat-network.eu/accessprevalencedata/keypublichealthindicators (accessed on 21 July 2014).

15. Christianson, A.; Howson, C.P.; Modell, B. March of Dimes: Global Report on Birth Defects, the Hidden Toll of Dying and Disabled Children; March of Dimes Birth Defects Foundation: New York, NY, USA, 2006. 
16. Vrijheid, M.; Martinez, D.; Manzanares, S.; Dadvand, P.; Schembari, A.; Rankin, J.; Nieuwenhuijsen, M. Ambient air pollution and risk of congenital anomalies: A systematic review and meta-analysis. Environ. Health Perspect. 2011, 119, 598-606.

17. Nieuwenhuijsen, M.J.; Martinez, D.; Grellier, J.; Bennett, J.; Best, N.; Iszatt, N.; Vrijheid, M.; Toledano, M.B. Chlorination disinfection by-products in drinking water and congenital anomalies: Review and meta-analyses. Environ. Health Perspect. 2009, 117, 1486-1493.

18. Padula, A.M.; Tager, I.B.; Carmichael, S.L.; Hammond, S.K.; Lurmann, F.; Shaw, G.M. The association of ambient air pollution and traffic exposures with selected congenital anomalies in the San Joaquin Valley of California. Amer. J. Epidemiol. 2013, 177, 1074-1085.

19. Padula, A.M.; Tager, I.B.; Carmichael, S.L.; Hammond, S.K.; Yang, W.; Lurmann, F.; Shaw, G.M. Ambient air pollution and traffic exposures and congenital heart defects in the San Joaquin Valley of California. Paediatr. Perinat. Epidemiol. 2013, 27, 329-339.

20. Padula, A.M.; Tager, I.B.; Carmichael, S.L.; Hammond, S.K.; Yang, W.; Lurmann, F.W.; Shaw, G.M. Traffic-related air pollution and selected birth defects in the San Joaquin Valley of California. Birth Defects Res. Pt. A 2013, 97, 730-735.

21. Agay-Shay, K.; Friger, M.; Linn, S.; Peled, A.; Amitai, Y.; Peretz, C. Air pollution and congenital heart defects. Environ. Res. 2013, 124, 28-34.

22. Schembari, A.; Nieuwenhuijsen, M.J.; Salvador, J.; de Nazelle, A.; Cirach, M.; Dadvand, P.; Beelen, R.; Hoek, G.; Basagaña, X.; Vrijheid, M. Traffic-related air pollution and congenital anomalies in Barcelona. Environ. Health Perspect. 2014, 122, 317-323.

23. Gianicolo, E.A.; Mangia, C.; Cervino, M.; Bruni, A.; Andreassi, M.G.; Latini, G. Congenital anomalies among live births in a high environmental risk area-A case-control study in Brindisi (southern Italy). Environ. Res. 2014, 128, 9-14.

24. Hwang, B.F.; Jaakkola, J.J.K. Ozone and other air pollutants and risk of oral clefts. Environ. Health Perspect. 2008, 116, 1411-1415.

25. Liberati, A.; Altman, D.G.; Tetzlaff, J.; Mulrow, C.; Gøtzsche, P.C.; Ioannidis, J.P.; Clarke, M.; Devereaux, P.J.; Kleijnen, J.; Moher, D. The PRISMA statement for reporting systematic reviews and meta-analyses of studies that evaluate health care interventions: Explanation and elaboration. PLoS Med. 2009, 6, doi:10.1136/bmj.b2700.

26. Warren, J.; Fuentes, M.; Herring, A.; Kanglois, P. Bayesian spatial-temporal model for cardiac congenital anomalies and ambient air pollution risk assessment. Environmetrics 2012, 23, 673-684.

27. Higgins, J.P.; Thompson, S.G.; Deeks, J.J.; Altman, D.G. Measuring inconsistency in meta-analyses. BMJ 2003, 327, 1557-1560.

28. Dadvand, P.; Rankin, J.; Rushton, S.; Pless-Mulloli, T. Ambient air pollution and congenital heart disease: A register-based study. Environ. Res. 2011, 111, 435-441.

29. Dadvand, P.; Rankin, J.; Rushton, S.; Pless-Mulloli, T. Association between maternal exposure to ambient air pollution and congenital heart disease: A register-based spatiotemperal analysis. Amer. J. Epidemiol. 2011, 173, 171-182.

30. Marshall, E.G.; Harris, G.; Wartenberg, D. Oral cleft defects and maternal exposure to ambient air pollutants in New Jersey. Birth Defects Res. Pt. A 2010, 88, 205-215. 
31. Dolk, H.; Armstrong, B.; Lachowycz, K.; Vrijheid, M.; Rankin, J.; Abramsky, L.; Boyd, P.A.; Wellesley, D. Ambient air pollution and risk of congenital anomalies in England, 1991-1999. Occup. Environ. Medicine 2010, 67, 223-227.

32. Hansen, C.A.; Barnett, A.G.; Jalaludin, B.B.; Morgan, G.G. Ambient air pollution and birth defects in Brisbane, Australia. PLoS One 2009, 4, doi:10.1371/journal.pone.0005408

33. Rankin, J.; Chadwick, T.; Natarajan, M.; Howel, D.; Pearce, M.S.; Pless-Mulloli, T. Maternal exposure to ambient air pollutants and risk of congenital anomalies. Environ. Res. 2009, 109, 181-187.

34. Strickland, M.J.; Klein, M.; Correa, A.; Reller, M.D.; Mahle, W.T.; Riehle-Colarusso, T.J.; Botto, L.D.; Flanders, W.D.; Mulholland, J.A.; Siffel, C,; et al. Ambient air pollution and cardiovascular malformations in Atlanta, Georgia, 1986-2003. Amer. J. Epidemiol. 2009, 169, 1004-1014.

35. Kim, O.J.; Ha, E.H.; Kim, B.M.; Seo, J.H.; Park, H.S.; Jung, W.J.; Lee, B.E.; Suh, Y.J.; Kim, Y.J.; Lee, J.T.; et al. $\mathrm{PM}_{10}$ and pregnancy outcomes: A hospital-based cohort study of pregnant women in Seoul. J. Occup. Environ. Med. 2007, 49, 1394-1402.

36. Giloba, S.M.; Mendola, P.; Olshan, A.F.; Langlois, P.H.; Savitz, D.A.; Loomis, D.; Herring, A.H.; Fixler, D.E. Relation between ambient air quality and selected birth defects, seven county study, Texas, 1997-2000. Amer. J. Epidemiol. 2005, 162, 238-252.

37. Institut de Veille Sanitaire (InVS). Available online: http://www.invs.sante.fr/fr/ Espace-presse/Communiques-de-presse/2014/L-Institut-de-veille-sanitaire-publie-pour-la-premierefois-des-estimations-nationales-sur-le-nombre-d-anomalies-congenitales-en-France (accessed on 18 July 2014).

38. Botto, L.D.; Lin, A.E.; Riehle-Colarusso, T.; Malik, S.; Correa, A.; National Birth Defects Prevention Study. Seeking causes: Classifying and evaluating congenital heart defects in etiologic studies. Birth Defects Res. Pt. A 2007, 79, 714-727.

39. Hackshw, A.; Rodeck, C.; Boniface, S. Maternal smoking in pregnancy and birth defects: A systematic review based on 173,687 malformed cases and 11.7 million controls. Hum. Reprod. Update. 2011, 17, 589-604.

40. Cresci, M.; Foffa, I.; Ait-Ali, L.; Pulignani, S.; Gianicolo, E.A.; Botto, N.; Picano, E.; Andreassi, M.G. Maternal and paternal environmental risk factors, metabolizing GSTM1 and GSTT1 polymorphisms, and congenital heart disease. Amer. J Cardiol. 2011, 108, 1625-1631.

41. Loane, M.; Dolk, H.; Morris, J.K.; EUROCAT Working Group. Maternal age-specific risk of non-chromosomal anomalies. BJOG 2009, 116, 1111-1119.

42. De la Vega, A.; López-Cepero, R. Seasonal variations in the incidence of some congenital anomalies in Puerto Rico based on the timing of conception. P. R. Health Sci. J. 2009, 28, 121-125.

43. Varela, M.M.; Nohr, E.A.; Llopis-González, A.; Andersen, A.M.; Olsen, J. Socio-occupational status and congenital anomalies. Eur. J. Public Health 2009, 19, 161-167.

44 Wells, G.A.; Shea, B.; O’Connell, D.; Peterson, J.; Welch, V.; Losos, M.; Tugwell, P. The Newcastle-Ottawa Scale (NOS) for Assessing the Quality of Nonrandomised Studies in Meta-Analyses. Ottawa Hospital Research Institute. 2014. Available online: http://www.ohri.ca/ programs/clinical_epidemiology/oxford.asp (accessed on 21 July 2014). 
45 Detsky, A.S.; Naylor, C.D.; O’Rourke, K.; McGeer, A.J.; L’Abbé, K.A. Incorporating variations in the quality of individual randomized trials into meta-analysis. J. Clin. Epidemiol. 1992, 45, 255-265.

46 Julian, P.T.H.; Sally, G. Cochrane Handbook for Systematic Reviews of Interventions, Version 5.1.0; The Cochrane Collaboration, 2011. Available online: www.cochrane-handbook.org (accessed on 21 July 2014).

47 Kampa, M.; Castanas, E. Human health effects of air pollution. Environ. Pollut. 2008, 151, 362-367.

48 Kannan, S.; Misra, D.P.; Dvonch, J.T.; Krishnakumar, A. Exposures to airborne particulate matter and adverse perinatal outcomes: A biologically plausible mechanistic framework for exploring potential effect modification by nutrition. Environ. Health Perspect. 2006, 114, 1636-1642.

49 Slama, R.; Darrow, L.; Parker, J.; Woodruff, T.J.; Strickland, M.; Nieuwenhuijsen, M.; et al. Meeting report: Atmospheric pollution and human reproduction. Environ. Health Perspect. 2008, 116, 791-798.

50 Baccarelli, A.; Bollati, V. Epigenetics and environmental chemicals. Curr. Opin. Pediatr. 2009, 21, 243-251.

51 Hou, L.; Wang, D.; Baccarell, A. Environmental chemicals and microRNAs. Mutat. Res. 2011, 714, 105-112.

(C) 2014 by the authors; licensee MDPI, Basel, Switzerland. This article is an open access article distributed under the terms and conditions of the Creative Commons Attribution license (http://creativecommons.org/licenses/by/3.0/). 